\title{
Ultraviolet variability of the Seyfert 1 galaxy Fairall $9^{\star}$
}

\author{
M.C. Recondo-González ${ }^{1}$, W. Wamsteker ${ }^{1,6}$, J. Clavel $^{2,6}$, P.M. Rodríguez-Pascual ${ }^{1,6}$, R. Vio ${ }^{3}$, Wang \\ Ting-gui ${ }^{1,5}$, M. Santos-Lleó ${ }^{4}$, and F. Makino ${ }^{7}$ \\ 1 ESA IUE Observatory, Apartado 50727, 28080-Madrid, Spain \\ 2 ISO Observatory, Astrophysics Division of ESA, Apartado 50727, 28080-Madrid, Spain \\ 3 Astronomy department, University of Padua, Vicolo del Osservatorio 5, 35122 Padva, Italy \\ 4 LAEFF, Apartado 50727, 28080-Madrid, Spain \\ ${ }^{5}$ Center for Astrophysics, University of Science and Technology of China, Hefei, 230026-Anhui, P.R. China \\ 6 Affiliated to the Astrophysics Division, Space Science Department, Noordwijk, The Netherlands \\ 7 Institute of Space and Astronautical Science, 3-1-1,Yoshinda, Sagamihara-shi, Kanagawa 229, Japan
}

Received February 29; accepted June 20, 1996

\begin{abstract}
The large amplitude of the variations of the Seyfert I Galaxy Fairall-9 between 1978 and 1991 make this Active Galaxy especially suitable for a combined study in terms of reverberation analysis and photoionization modeling over the velocity field of the broad lines of its ultraviolet spectrum. We have combined our ultraviolet data with those available at other wavelengths to derive the intrinsic ionizing continuum and to compare the predictions of the photoionization models with the observations. The UV continuum varies with a factor of $33 \pm 4$ on a characteristic time scale of 182 days. The intrinsic spectral index UV-optical is $\alpha=-0.07 \pm 0.02$ and the optical variations do not lag behind the UV variations at the mean sampling interval of 96 days. In the near IR, the $J$ band flux presents a direct extension of the UV-optical continuum $\left(\alpha_{J-\mathrm{UV}}=-0.07 \pm 0.05\right)$. Only at low flux levels is the $2-10 \mathrm{keV}$ flux proportional to the UV continuum, at higher UV flux levels proportionality between the $\mathrm{X}$-rays and the UV brightness breaks down. The continuum spectral energy distribution (SED) of the nucleus supports the previously reported FIR-NIR excess associated with $0.02 M_{\odot}$ dust at a distance of some $400 \pm 100$ light-days, as well as a soft X-ray excess, possibly associated with the reprocessing on an accretion disk of the hard X-rays emitted from a region above the disk. The presence of strong $\mathrm{Fe} \mathrm{K} \alpha$ line in the GINGA spectra of this galaxy does lend support to this model. Line profile variability has been used to isolate four gaussian line components, which are sufficient to describe all lines at all levels of brightness in a consistent way: one narrow (i.e. unresolved at the IUE resolution), and three broad components: a central (velocity same as the nar-

Send offprint requests to: G. Wuchterl

* Tables 1, 10, 11, 12, 13 only available in electronic form at CDS via anonymous ftp to CDSARC.U-strasbg.fr (130.79.128.5) or via

http://cdsweb.u-strasbg.fr/Abstract.html
\end{abstract}

row line), a red shifted $\left(v=3300 \mathrm{~km} \mathrm{~s}^{-1}\right)$ and a blue shifted $\left(v=-3600 \mathrm{~km} \mathrm{~s}^{-1}\right)$ one. The three broad components are strongly correlated with the UV continuum, indicating that photoionization is the dominant mechanism in the BLR. Correlation analysis shows different delays between the broad components and the UV continuum: respectively, $400 \pm 100,-4 \pm 70$ and $230 \pm 95$ days for the central, red and blue one. Only the red component of Ly $\alpha \lambda 1216$ and CIV $\lambda 1549$ appear to vary linearly with the continuum and give significant transfer functions. The resulting transfer functions are peaked at zero days delay and are unresolved at the average time resolution (96 days) of our data. The photoionization models (CLOUDY) applied to the line ratios in these components, indicate that the Broad-Line-Region (BLR) is situated between $50-250$ light-days from the ionizing source, with an hydrogen density of $10^{12-10} \mathrm{~cm}^{-3}$, a column density of $10^{23-26} \mathrm{~cm}^{-2}$ and a covering factor of $12-2 \%$, with an ionization parameter between 0.003 and 0.089 . However, no optically thick model reproduces the Ly $\alpha /$ CIV and Ly $\alpha /$ NV ratios. From these results we propose a model for the structure and dynamics of the BLR: the mass of the central compact object is $M \approx 210^{8} M_{\odot}$. Around this exist two distinct gas zones within the BLR: the gas producing the central component at $\approx 200$ light-days and gas, emitting the red and the blue components, at $\approx 80$ light-days moving inward to a central source. These results require that both these gas zones be localized along the line-of-sight or, alternatively, that the continuum emission must be strongly anisotropic. Besides, the gas emitting the central component is most likely mixed with the dust and the central gas to dust mass ratio is Mass (central gas) $/$ mass(dust) $=100-750$.

Key words: galaxies: individual (Fairall 9) — galaxies: Seyfert - ultraviolet: spectra 


\section{Introduction}

Fairall-9 (F-9 = ESO 113-IG45) is a Seyfert 1 galaxy $\left(m_{V} \approx 13 \mathrm{mag}\right)$ in the Southern Hemisphere $(\alpha(1950)=$ $01^{\mathrm{h}} 21^{\mathrm{m}} 51^{\mathrm{s}} .22$ and $\left.\delta(1950)=-59^{\circ} 0.3^{\prime} 58^{\prime \prime} \cdot 9\right)$ at $z=0.0461$ (Ward \& Wilson 1978). It was discovered spectroscopically as a Seyfert 1 galaxy by Fairall (1977), who noted its high luminosity of $M_{V} \approx-24$ and classified it as an extreme Seyfert 1 galaxy, almost a QSO (Fairall 1977; Ward \& Wilson 1978; West et al. 1978). Its morphology shows an unresolved nucleus $\left(<1^{\prime \prime}=660 \mathrm{pc}\right.$ at $140 \mathrm{Mpc}$, taking $\left.H_{0}=100 \mathrm{~km} \mathrm{~s}^{-1} \mathrm{Mpc}^{-1}\right)$ with an envelope with several spiral arms extending until $\approx 20^{\prime \prime}$ from the center and an elliptical companion galaxy at $28^{\prime \prime} \mathrm{SE}$. The diameter of the outer envelope is $64^{\prime \prime}(40 \mathrm{kpc})$, and a jet is suggested to exist out to $\approx 55 \mathrm{kpc} \mathrm{SE}$ of the nucleus (West et al. 1978). Griersmith \& Visvanathan (1979) classified it as an early type spiral galaxy, but Véron-Cetty et al. (1991) consider it an elliptical giant galaxy. Its optical linear polarization is low, $p=0.4 \pm 0.11 \%$, as in most Seyfert galaxies, consistent with interstellar polarization by dust from our galaxy only (Martin et al. 1983).

F-9 has displayed an unusually large amplitude in its variability: it decreased in brightness from 1978 to 1984 with $\Delta m_{V}=0.8 \mathrm{mag}$ and in the UV with $\Delta m(1350 \AA)=$ $3.6 \mathrm{mag}$, corresponding to maximum to minimum brightness ratio $R_{\max }=27$ (Wamsteker et al. 1985) and increased afterwards, but never recovered its discovery brightness. Similar variations have been observed in the NIR (Glass 1986; Clavel et al. 1989) and X-rays (Morini et al. 1986), but with smaller amplitude than that seen in the UV. The optical variations between 1979 and 1990 have been extensively documented by Lub \& De Ruiter (1992). More recent X-ray observations have been made with GINGA (Makino, private communication) and ROSAT (Walter et al. 1995). FIR observations with IRAS have been reported by Edelson \& Malkan (1987) and radio observations by Véron-Cetty et al. (1991).

The emission lines have been studied in considerable detail. The narrow lines of $[\mathrm{OIII}] \lambda \lambda 4959,5007$, have been suggested to show a blue asymmetry in the profile (Whittle 1985; Busko \& Steiner 1988, 1989) and no flux variability (Kollatschny \& Fricke 1985; Wamsteker et al. 1985; Lub \& De Ruiter 1992). On the other hand, the intensity and profiles of the broad lines are highly variable. The broad UV lines (Ly $\alpha \lambda 1216$, CIV $\lambda 1549$ and $\operatorname{MgII} \lambda 2798$ ) have been extensively studied by Chapman et al. (1985), Wamsteker \& Colina (1986) and Clavel et al. (1989), and the optical lines by Wamsteker et al. (1985) and Lub \& De Ruiter (1992). The complex structure of the UV line profiles has been discussed by O'Brien \& Zheng (1990) and Zheng \& O'Brien (1990). A detailed profile decomposition by means of gaussian components has been made for $\mathrm{H} \beta \lambda 4861$ by Wamsteker et al. (1985). Cross correlation studies of the total intensity of Ly $\alpha \lambda 1216$, CIV $\lambda 1549$ and MgII $\lambda 2798$ showed a delay in the line re- sponse to UV continuum variation of $155 \pm 45$ days for all three lines (Clavel et al. 1989). Koratkar \& Gaskell (1989) suggest that CIV seems to show evidence of infall motion, while MgII $\lambda 2798$ suggests random motion. Photoionization model calculations on the total line intensities by Clavel \& Santos-Lleó (1990) indicate a BroadLine-Region (BLR) at 100 light-days from the ionizing source, which needs a cut-off energy of 0.83 ryd in the big blue bump, a density of $10^{9.5-11} \mathrm{~cm}^{-3}$ and a column density of $10^{23.5} \mathrm{~cm}^{-2}$ to be able to explain the WamstekerColina effect, the apparent saturation of CIV at high luminosities as defined by Shields et al. (1995). Binette et al. (1989) require a similar cut-off energy at 0.73 ryd using a different photoionization code.

We discuss here all observations made in the UV (Sect. 2) and compare these with the observations made at other wavelengths to derive the Spectral Energy Distribution (SED) in Sect. 3. In Sect. 4 we give a detailed analysis of the emission line variability, including line decomposition. Section 5 combines the results of Sects. 3 and 4 to compare the data with photoionization calculations and derive the nature of the Broad-Line-Region (BLR) in Sects. 6 and 7. A summary of the results is given in Sect. 8 .

\section{Ultraviolet observations}

The UV spectra have been taken with the International Ultraviolet Explorer (IUE) satellite (Boggess et al. 1978a, b, 1989; Fälker et al. 1989). We have used all spectra taken between 1978 and 1991 from the IUE Archive, many of these have been taken under the ESA IUE Observatory Programs. In these 13 years (4903 days) 158 spectra were obtained through the large aperture (LAP: $\left.10^{\prime \prime} \times 20^{\prime \prime}\right)$ in low resolution mode $(6-8 \AA)$ ), 90 with the SWP (Short Wavelength Prime) camera between 1150-1950 A, and 68 with the LWR/P (Long Wavelength Redundant/Prime) cameras between $1900-3200 \AA$. All were reduced with the most recent version of the IUE Image Processing System (IUESIPS: Clavel 1987). To adjust for shifts in the wavelength scale due to centering uncertainties in the LAP, all spectra were aligned by centering the peak of Ly $\alpha \lambda 1216$ at $\lambda$ (observed) $=1271.6 \AA$ and for $\operatorname{MgII} \lambda 2798$ at $\lambda$ (observed) $=2929.08 \AA$. The LWR spectra were corrected for the sensitivity loss of this camera with time (Clavel et al. 1988). On the other hand, because the sensitivity degradation of the SWP and LWP Cameras is small (some $13 \%$ over the 13 years) and shows a smooth variation with wavelength above $1200 \AA$ (Sonneborn \& Garhart 1987; Bohlin \& Grillmair 1988), its effect on the line shape and continuum variation is very small in comparison to the overall variability of F-9 and no correction was applied for it. The IUE Fine-Error-Sensor (FES) allows optical photometry in a $12^{\prime \prime}$ equivalent square aperture with an effective wavelength for AGN-type energy distributions of $\lambda \approx 5200 \AA$. The 58 FES measurements have been corrected for the sensitivity loss with time. Due to FES 
reference point (the position on the detector where the photometric measurements are made) changes, two different corrections had to be applied: one between 1980 and 1989 (Pérez et al. 1991) and another after 1990 (Pérez 1991).

Rather than listing here again the details for the individual spectra, we refer the reader for the observational details to Clavel et al. (1989) and Courvoisier \& Paltani (1992). In most cases the IUE spectra were exposed optimally for Ly $\alpha \lambda 1216$ and $\operatorname{MgII} \lambda 2798$ but those overexposed in the lines (saturation in more than 5 pixels) have not been used for the line decomposition study (SWP 1804, 2215, 4539, 13351, 16559, 16890, 17249, 17446, 17521, 18094, 18891, 21261, 21286, 25825, 26774, 27007, 27008, 27400, 28213, 28307, 28632, 28633, 29545, 29991, 30160, 31176, 34053, 37548, 40179; LWP 2037, 5872, 6201, $6491,8081,8562,9426)$. Table 1 gives the UV continuum fluxes and FES counts for the IUE data. Whenever there are several spectra and several FES measurements the values in Table 1 give the mean value and the rms error for the fluxes and the FES counts. In the case of a single FES measurement, we have adopted an error of $6 \%$, corresponding to the average of the rms deviations of the individual measurements at the epochs when more than one measurement was available.

\section{The spectral energy distribution (SED) of F-9}

\subsection{Multi-wavelength continuum: corrections and measurements}

We have used the observations of F-9, taken between 1978 and 1991, at all wavelengths from the radio to X-rays to derive the nuclear continuum spectral energy distribution (SED), which will later be used in the photoionization models. The X-ray, UV, and optical lightcurves are shown in Fig. 1. All data were corrected for $E(B-V)=0.035$ which is derived from the combined hydrogen column density from our Galaxy and the Magellanic Stream of $N_{\mathrm{H}}=1.910^{20} \mathrm{~cm}^{-2}$ (Morini et al. 1986; Clavel \& SantosLleó 1990). Using this color excess we applied Seaton's law (1979) to correct the ultraviolet and the optical. In the NIR we used the extinction law for this wavelength region from Rieke \& Lebofsky (1985). To evaluate the contribution in the FIR due to cold interstellar dust from the galaxy disk of F-9, we used the results for normal earlytype spiral galaxies from the IRAS observations derived by De Jong et al. (1984). The resulting disk flux of 120 and $370 \mathrm{mJy}$ at respectively 60 and $100 \mu \mathrm{m}$, has been subtracted from the IRAS fluxes for F-9 in these bands. The subtraction of the stellar contribution in the optical and the near IR will be discussed in Sect. 3.1.1. Unless otherwise specified, we will use observed wavelength in the following discussion.

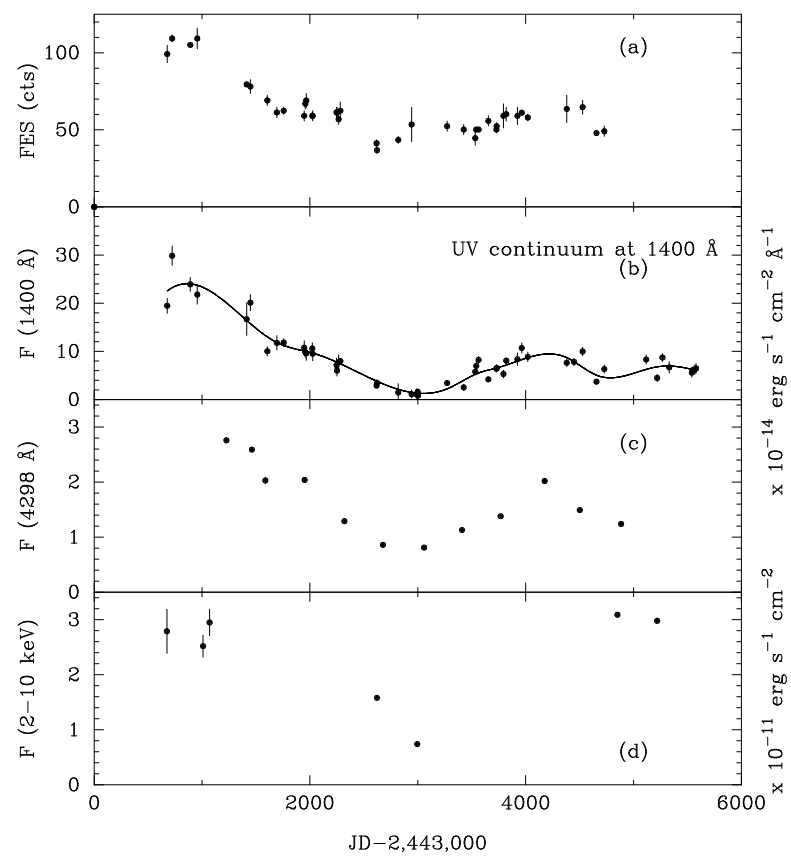

Fig. 1. Light curves of the UV, optical and $X$ rays continua. a) FES light curve $\left(\lambda_{\text {eff }} \approx 5200 \AA\right)$. b) $F(1400 \AA)$ light curve. The full drawn line is the cubic spline interpolation used to derive the UV fluxes at times when no simultaneous observations were available. c) $F(4298 \AA$ A ) light curve (Table 2). d) $F(2-10 \mathrm{keV}$ ) light curve (Table 3$)$. N.B. At minimum $(\mathrm{JD} \approx 3000)$ the object was too faint to be detected with the FES

\subsubsection{The ultraviolet and optical}

The ultraviolet continuum fluxes in Table 1 have been measured in three "line-free" windows centered at 1171 (1158-1184), 1400 (1390-1410) and 1910 (1895-1925) $\AA$, and are corrected for $E(B-V)=0.035$ extinction. When more than one spectrum was available on the same day, both fluxes and errors have been averaged. Since $F(1171 \AA)$ is rather noisy -it is measured at the short wavelength side of the geocoronal emission- and $F(1910 \AA)$ already has a small contribution of the Balmer continuum and the FeII emission (Small Blue Bump), both present above $\approx 1500 \AA$ (rest wavelength), we will use the $F(1400 \AA)$ as reference UV continuum. For those cases where the data in the UV and at other wavelengths are not exactly simultaneous, we use an interpolation of the UV light curve fitted with a cubic spline using a smoothing factor of $\chi^{2}$ (reduced) $=3.75$ as shown in Fig. 1b to the epoch of the non-UV observation. Table 1 also gives the FES counts corrected for $E(B-V)=0.035$. Groundbased optical photometry between 1979-1990 of F-9 has also been reported by Lub \& De Ruiter (1992). We have corrected their $B$ band fluxes at $4298 \AA$ measured with an aperture radius of $55^{\prime \prime} 8$ for $E(B-V)=0.035$ (versus $E(B-V) \approx 0.02$ as used by them; De Ruiter, private 
communication). These values are given in Table 2 together with the corresponding UV fluxes, derived from the interpolated ultraviolet lightcurve. Both the FES and the $B$ lightcurve are shown together with $F(1400 \AA)$ and $\mathrm{X}$-rays in Fig. 1.

\subsubsection{The stellar contribution to the optical continuum}

The FES light curve (Fig. 1) is very similar to the UV, but its amplitude, as is common in Seyfert I galaxies, is considerably smaller with $R_{\max }=3.0 \pm 0.2$. The Cross Correlation Function (CCF; Gaskell \& Peterson 1987) of the FES counts with $F(1400 \AA)$ peaks at $-4 \pm 76$ days (Fig. 2c), indicating that there is no delay between the variations at these wavelengths. As a consequence of this both fluxes are highly correlated (Fig. 3c):

$$
\begin{aligned}
& \operatorname{FES}=(2.76 \pm 0.13) \times F_{1400 \AA}{ }^{+} \\
& +(34.91 \pm 1.12)(\text { counts }) .
\end{aligned}
$$

$\left(F_{1400 \AA}\right.$ is in units of $\left.10^{-14} \mathrm{erg} \mathrm{s}^{-1} \mathrm{~cm}^{-2} \AA^{-1}\right)$ with $r=0.938$. Using the calibration of Imhoff \& Wasatonic (1986) one can in an approximate way convert the FES counts to Johnson $V$-band fluxes. Using a color index of $(B-V) \approx 0.2$ for the total central flux of F-9 (Griersmith \& Visvanathan 1979) we obtain $\mathrm{FES}_{V}\left(10^{-14} \mathrm{erg} \mathrm{s}^{-1} \mathrm{~cm}^{-2} \AA^{-1}\right)=0.024 \times \mathrm{FES}($ cts $)$, changing relation (1) into:

$$
\begin{aligned}
& \mathrm{FES}_{V}=(0.066 \pm 0.007) \times F_{1400 \AA} \AA^{+} \\
& +(0.838 \pm 0.083)\left(10^{-14} \mathrm{erg} \mathrm{s}^{-1} \mathrm{~cm}^{-2} \AA^{-1}\right) .
\end{aligned}
$$

Here we have added the estimated error of $6 \%$, associated with the calibration used (Imhoff \& Wasatonic 1986), to the correlation errors. Both relations (1 and 2) show a nonnegligible constant term most likely due to the stellar contribution from the central bulge of the underlying galaxy. This contribution dilutes the intrinsic nuclear variation of the AGN itself and causes the smaller amplitude of the optical variations with respect to the UV. Similar results are obtained from the optical data of Lub \& De Ruiter (1992) and their light curve is also very similar to the UV one (Fig. 1c) and its amplitude, $R_{\max }=3.41 \pm 0.06$, is similar to that of the FES. The $C C F$ of $F(4298 \AA)$ with $F(1400 \AA)$ peaks at $-16 \pm 93$ days (Fig. 2c), confirming the strict simultaneity at the time resolution of 96 days of the variations at these wavelengths. Linear correlation gives (Fig. 3d):

$$
\begin{aligned}
& F_{4298 \AA}=(0.115 \pm 0.001) \times F_{1400 \AA}+ \\
& +(0.670 \pm 0.008)\left(10^{-14} \mathrm{erg} \mathrm{s}^{-1} \mathrm{~cm}^{-2} \AA^{-1}\right) .
\end{aligned}
$$

$r=0.969$, using the interpolated $F(1400 \AA)$ light curve. Also here the non-zero constant term is most likely due to the stellar contribution and is the main cause for the smaller amplitude of the $F(4298 \AA)$ variations compared to the UV. The constant terms in these relations causes the apparent hardening of the UV-optical spectral index $\alpha\left(F_{\nu} \propto \nu^{+\alpha}\right.$; e.g. Table 2 for $\left.F(4298 \AA)\right)$ with increasing UV brightness. Since we want to use the observed AGN SED later as input spectrum for the photoionization models at different brightness levels, we need to correct the SED for these contributions. The constant term in Eqs. (2) and (3) is dominated by the stellar contribution, however many optical FeII multiplets and the forbidden lines of [OIII] $\lambda 5007,4959$ as well as $\mathrm{H} \beta \lambda 4861$ contribute both to the FES counts and $F(4298 \AA)$. To evaluate the Fe II contribution, we use the results of Wamsteker et al. (1985), deriving a value of $\approx 0.110^{-14} \mathrm{erg} \mathrm{s}^{-1} \mathrm{~cm}^{-2} \AA^{-1}$, from high resolution spectra and FeII model fitting. Subtracting this from the constant in Eq. (2) we obtain $(0.7 \pm 0.1) 10^{-14} \mathrm{erg} \mathrm{s}^{-1}$ $\mathrm{cm}^{-2} \AA^{-1}$ (for the $V$ band). Similarly we estimated the FeII contributing to the $F(4298 \AA)$, using the same FeII model and the bandpass of this band (Lub \& Pel 1977). This resulted in $\approx 0.0610^{-14} \mathrm{erg} \mathrm{s}^{-1} \mathrm{~cm}^{-2} \AA^{-1}$. Subtracting this from the constant in relation (3) gives $(0.61 \pm 0.01) 10^{-14} \mathrm{erg} \mathrm{s}^{-1} \mathrm{~cm}^{-2} \AA^{-1}$ (for the $F(4298 \AA)$ ). In the absence of optical spectra or images, taken specifically for this purpose, we can compare the results of this procedure with other independent determinations of the stellar contribution to the nuclear flux in those wavelengths were it is important. Griersmith \& Visvanathan (1979) used a De Vaucouleurs law ( $r^{1 / 4}$ distribution) for the brightness distribution, which they combined with their measured brightness distribution to calculate the stellar galaxy flux in $V$ for any aperture. For the FES (taken in $V$ ) with an aperture radius of 6.77 , we obtain $(0.50 \pm 0.21) 10^{-14} \mathrm{erg} \mathrm{s}^{-1} \mathrm{~cm}^{-2} \AA^{-1}$, consistent with the above. For the $F(4298 \AA)$ aperture radius of 5 !' 8 , and taking the color index for the stellar population in the galaxy $(B-V)=0.72 \pm 0.12 \mathrm{mag}$ (Griersmith \& Visvanathan 1979), we obtain (taking $B=4298 \AA$ ) $(0.47 \pm 0.24) 10^{-14} \mathrm{erg} \mathrm{s}^{-1} \mathrm{~cm}^{-2} \AA^{-1}$, also in good agreement with the above. Since the stellar emission of the galaxy estimated by Griersmith \& Visvanathan (1979) is in good agreement with those derived by us we will use it in what follows. This value is approximately half of that derived for $F(4298 \AA)$ by Lub \& De Ruiter (1992), but they used a disk-like exponential brightness distribution and may have underestimated the contributions due to FeII considerably. A disk distribution might not be appropriate for the apertures used by Lub \& de Ruiter $\left(5{ }^{\prime \prime} 8-14\right.$.' 05$)$, because Griersmith \& Visvanathan (1979) suggest that the nuclear bulge extends out to $11^{\prime \prime} 75$. Véron-Cetty et al. (1991) find also that a $r^{1 / 4}$ distribution gives a good representation of their observations of the galaxy surface brightness distribution for F-9. With their value of $M_{V}$ and $(B-V)=0.75 \mathrm{mag}$ for the galaxy, we derive the expected contribution to the FES counts to be $\approx 0.41-0.4510^{-14} \mathrm{erg} \mathrm{s}^{-1} \mathrm{~cm}^{-2} \AA^{-1}$ and to the $F(4298 \AA) \approx 0.37-0.4110^{-14} \mathrm{erg} \mathrm{s}^{-1} \mathrm{~cm}^{-2} \AA^{-1}$. Since these independent estimates of the stellar 
Table 2. Ultraviolet and optical continuum

\begin{tabular}{|c|c|c|c|c|}
\hline $\begin{array}{c}\text { JD } \\
-2443000 \\
\end{array}$ & $\begin{array}{c}F_{1400} \AA^{\text {(interpolated) }} \\
10^{-14} \mathrm{erg} \mathrm{s}^{-1} \\
\end{array}$ & $\begin{array}{ll} & F_{4298 \AA} \\
-2 & \AA^{-1} \\
\end{array}$ & $\alpha_{1400-4298 \AA}$ & $\begin{array}{c}\text { Balmer C. }(3837 \AA) \\
10^{-14} \mathrm{erg} \mathrm{s}^{-1} \mathrm{~cm}^{-2} \AA^{-1}\end{array}$ \\
\hline 1226 & 20.06 & $2.76 \pm 0.03$ & $-0.232 \pm 0.022$ & $0.96 \pm 0.07$ \\
\hline 1462 & 15.48 & $2.59 \pm 0.03$ & $-0.406 \pm 0.024$ & $1.46 \pm 0.06$ \\
\hline 1587 & 13.28 & $2.03 \pm 0.06$ & $-0.326 \pm 0.061$ & $0.88 \pm 0.10$ \\
\hline 1951 & 10.04 & $2.04 \pm 0.04$ & $-0.579 \pm 0.040$ & $1.32 \pm 0.06$ \\
\hline 2320 & 7.00 & $1.29 \pm 0.01$ & $-0.492 \pm 0.016$ & $0.57 \pm 0.10$ \\
\hline 2677 & 3.24 & $0.86 \pm 0.02$ & $-0.817 \pm 0.048$ & $0.40 \pm 0.01$ \\
\hline 3059 & 1.26 & $0.81 \pm 0.01$ & $-1.606 \pm 0.025$ & $0.56 \pm 0.03$ \\
\hline 3410 & 3.80 & $1.13 \pm 0.01$ & $-0.919 \pm 0.018$ & $0.78 \pm 0.01$ \\
\hline 3768 & 6.78 & $1.38 \pm 0.01$ & $-0.581 \pm 0.015$ & $0.72 \pm 0.03$ \\
\hline 4176 & 9.42 & $2.02 \pm 0.05$ & $-0.627 \pm 0.051$ & $1.20 \pm 0.11$ \\
\hline 4502 & 7.05 & $1.492 \pm 0.002$ & $-0.616 \pm 0.003$ & $0.86 \pm 0.01$ \\
\hline 4885 & 4.75 & $1.24 \pm 0.02$ & $-0.803 \pm 0.033$ & $0.80 \pm 0.03$ \\
\hline
\end{tabular}

NOTE: The optical data are taken from Lub \& De Ruiter (1992). Balmer C. discussed in 4.1.

contribution to the optical measurements of the nuclear brightness are all consistent within the measurement uncertainties, we can be confident that all constant components in the observations of the nuclear region of F-9 are properly allowed for in the derivation of the SED of the active galactic nucleus. Then, using the slopes of the relations (2) and (3), we obtain $\alpha(1400 \AA-\mathrm{FES}(\mathrm{V}))=$ $0.00 \pm 0.18$ and $\alpha(1400 \AA-4298 \AA)=-0.07 \pm 0.02$, equal within the errors. As the second value has a smaller error, we will use from now on a UV-optical spectral index $\alpha_{\mathrm{UV}-\mathrm{op}}=-0.07 \pm 0.02$. After correction for $E(B-V)=0.035$, instead of $E(B-V)=0.06$ used by Wamsteker et al. (1985), this value is similar to the index obtained from their Table $1, \alpha_{\mathrm{UV}-\mathrm{op}}=0.06 \pm 0.27$.

\subsubsection{The infrared}

Near infrared (NIR) observations of F-9 in $J(1.15 \mu \mathrm{m})$, $H(1.58 \mu \mathrm{m}), K(2.10 \mu \mathrm{m})$ and $L(3.35 \mu \mathrm{m})$ have been reported in Glass (1986) and in Clavel et al. (1989). From these data we only use those coinciding in temporal coverage with the UV observations and only the data with an aperture radius of $6^{\prime \prime}$ to avoid variations in stellar light contributions in the photometry. All fluxes have been corrected for $E(B-V)=0.035$.

Edelson \& Malkan (1987) report on four IRAS observations at $12,25,60$ and $100 \mu \mathrm{m}$ made over a period of 14 days, and find no significant variability in any of these bands. We use here for each band the mean flux and the rms of the individual measurements. The values for the mean epoch $(\mathrm{JD}-2443000)=2476$, are $380 \pm 20$, $550 \pm 20,590 \pm 40$ and $830 \pm 30 \mathrm{mJy}$ for $12,25,60$ and $100 \mu \mathrm{m}$, respectively. Subtracting the disk dust contribution at 60 and $100 \mu \mathrm{m}$ (120 and $370 \mathrm{mJy}$, respectively. See above), the fluxes for these two bands become $470 \pm 40$ and $460 \pm 30 \mathrm{mJy}$. Note the agreement between the $12 \mu \mathrm{m}$ IRAS point and the $\mathrm{N}_{3}$ measurement (also at $12 \mu \mathrm{m}$ ) taken $\approx 600$ days earlier (see Fig. 8 by Clavel et al. 1989), confirming that the $12 \mu \mathrm{m}$ radiation is not variable. The shape of the light curves in $J(1.15 \mu \mathrm{m}), H(1.58$
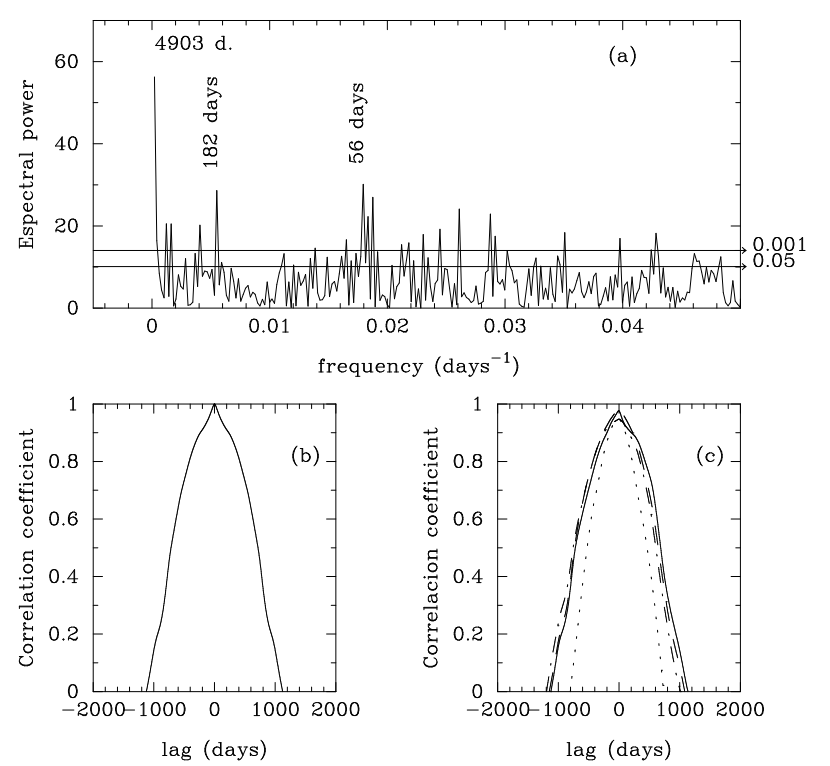

Fig. 2. Variability features of the UV-optical continuum. a) The power spectrum (Press \& Teukolsky 1988 algoritm) of the variations for $F(1400 \AA)$. The peak at 4903 days represents the total temporal range of the data and is not a real variability time scale. b) $A C F$ (Autocorrelation Function; Gaskell \& Peterson 1987) for $F(1400 \AA)$. c) $C C F$ for $F(1171 \AA$ ) (solid line), for $F(1910 \AA)$ (dashed line), FES counts (dot-dashed line) and $F(4298 \AA)$ (dotted line) with respect to $F(1400 \AA)$. The formal delay between these is respectively, $4 \pm 67,0 \pm 65$, $-4 \pm 76$, and $-16 \pm 93$ days

$\mu \mathrm{m}), K(2.10 \mu \mathrm{m})$ and $L(3.35 \mu \mathrm{m})$ are very similar to the UV (see Clavel et al. 1989), with a considerably smaller amplitude at $R_{\max }=1.98 \pm 0.08,2.53 \pm 0.10,2.88 \pm 0.11$ and $2.44 \pm 0.16$, respectively. Clavel et al. (1989) derived delays of respectively $-20 \pm 100,250 \pm 100,385 \pm 100$ and 


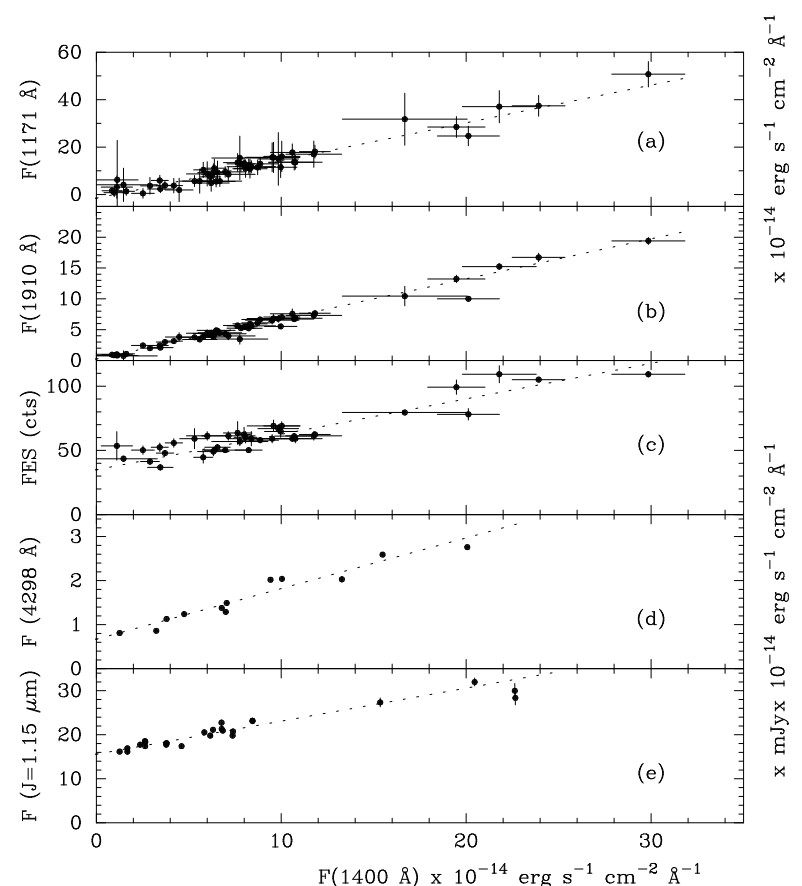

Fig. 3. Correlations between the continuum fluxes at different wavelengths versus the UV flux $F(1400 \AA)$. Linear regression lines are shown as dotted lines. a) $F(1171 \AA)$. b) $F(1910 \AA)$. c) $\operatorname{FES}$ counts $\left(\lambda_{\text {eff }} \approx 5200 \AA\right)$. d) $F(4298 \AA)$. e) $F_{J}(1.15 \mu \mathrm{m})$

$410 \pm 110$ days for $J, H, K$ and $L$, while we have obtained that the $J$ band and the UV fluxes are related by (Fig. 3e):

$$
\begin{aligned}
& F_{J}=(0.75 \pm 0.03) \times F_{1400 \AA}+(15.59 \pm 0.19)(\mathrm{mJy}) \\
& \left(F_{1400 \AA} \text { as } 10^{-14} \mathrm{erg} \mathrm{s}^{-1} \mathrm{~cm}^{-2} \AA^{-1}\right)
\end{aligned}
$$

with $r=0.950$, or, converting all units to $10^{-14} \operatorname{erg~s}^{-1} \mathrm{~cm}^{-2} \AA^{-1}$ :

$$
\begin{aligned}
& F_{J}=(0.0170 \pm 0.0007) \times F_{1400 \AA} \AA^{+} \\
& +(0.354 \pm 0.004)\left(10^{-14} \operatorname{erg~s}^{-1} \mathrm{~cm}^{-2} \AA^{-1}\right) .
\end{aligned}
$$

The constant term in relations (4) and (5) is again dominated by the stellar contribution, while also the $H$, $K$, and $L$ fluxes are affected, but to a progressively lesser extent.

The stellar emission in the near IR for F-9 with an aperture radius of $6^{\prime \prime}$, using the surface brightness distribution in $V$ (with an error of $3 \%$ ) of Griersmith \& Visvanathan (1979) and color indexes of $V-K=2.99$, $J-H=0.76 \pm 0.02, H-K=0.08 \pm 0.02$ and $K-L=$ $0.28 \pm 0.03$ (Clavel et al. 1989) is $13 \pm 6,17 \pm 7,11 \pm 4$ and $7 \pm 3 \mathrm{mJy}$ for $J, H, K$ and $L$, respectively. Glass (1986) comments that these color indexes are consistent with the population found in normal galaxies $(V-K=2.99$ is characteristic of giant and supergiant stars of K3-K4 spectral-type (Johnson 1966)). This stellar contribution at $J\left(13 \pm 6 \mathrm{mJy}=0.3 \pm 0.110^{-14} \mathrm{erg} \mathrm{s}^{-1} \mathrm{~cm}^{-2} \AA^{-1}\right)$ is similar, within the errors, to the constant term in Eqs. (4) or (5). Therefore, the behavior in the $J$-band can be fully understood in terms of a variable source, which is a direct extension of the UV-optical power-law, and a constant part due to the stellar contribution. Thus there appears to be no evidence for the presence of a steep constant IR power law, extending into the optical, as suggested by Edelson \& Malkan $(1986,1987)$ and Carleton et al. (1987). For the AGN (without the stellar contribution) we obtain an intrinsic $J$ - UV spectral index of $\alpha(\mathrm{UV}-J)=\alpha(1400 \AA-1.15 \mu \mathrm{m})=-0.07 \pm 0.05$, equal to the index obtained between 1400 and $4298 \AA$, confirming that the variable $J$ flux is a direct extension of the UV-optical.

\subsubsection{The X-rays}

Morini et al. (1986) have summarized the F-9 hard X-ray observations from 1970 to 1984 obtained with the Uhuru, Ariel 5, HEAO (1 and 2) and EXOSAT satellites. We use here again only data after 1978, for comparison with the UV continuum. Two GINGA observations on 1989 November 20-21 and in 1990 November 22-25 are included (Makino, private communication). The $2-10 \mathrm{keV}$ fluxes are listed in Table 3 together with the interpolated UV flux and the light curves are shown in Fig. 1d with the UV and the optical ones. The GINGA data give hard Xray spectral indexes of $\alpha=-0.89 \pm 0.05(\mathrm{JD}=4851)$ and $\alpha=-0.85 \pm 0.05(\mathrm{JD}=5219)$, both with a fixed column density of $N_{\mathrm{H}} \approx 310^{20} \mathrm{~cm}^{-2}$ and also show a strong $\mathrm{Fe}$ emission line centered at $6.64 \pm 0.24 \mathrm{keV}$, with an equivalent width of $360 \pm 140 \mathrm{eV}$ on $\mathrm{JD}=4851$ and $6.63 \pm 0.24 \mathrm{keV}$ and $360 \pm 140 \mathrm{eV}$ on $\mathrm{JD}=5219$. The only Soft X-ray observation has been taken with the ROSAT satellite at the same date as the second GINGA observation $(\mathrm{JD}=5219)$, simultaneous with one of the UV spectra (in the context of the RIASS program: De Martino et al. 1991). Walter et al. (1995) find that for a column density of $N_{\mathrm{H}}=2.8410^{20} \mathrm{~cm}^{-2}$ two power laws are required. So, he obtains for the GINGA spectrum a spectral index of $\alpha=-0.84$ and for the $2-10 \mathrm{keV}$ flux of $3.0410^{-11} \mathrm{erg} \mathrm{s}^{-1} \mathrm{~cm}^{-2}$ very similar to Makino's values, and for the ROSAT spectrum, $\alpha=-2.6$ and $F(0.15-2 \mathrm{keV})=2.210^{-11} \mathrm{erg} \mathrm{s}^{-1} \mathrm{~cm}^{-2}$, indicative of a strong soft X-ray excess.

\subsubsection{Radio}

The only radio observation reported for F-9 has been made at $4.7 \mathrm{GHz}$ (Véron-Cetty et al. 1991) with the Australian Telescope on 24 December, $1990(\mathrm{JD}=5249)$ and they give a $3 \sigma$ upper limit to the flux of 5 mJy, classifying F-9 as a weak radiosource. 
Table 3. Ultraviolet and X-rays continuum

\begin{tabular}{|c|c|c|c|}
\hline \hline $\begin{array}{c}\text { JD } \\
-2443000\end{array}$ & $\begin{array}{c}F_{1400 \AA} \AA^{\text {(interpolated) }} \\
10^{-14} \mathrm{erg} \mathrm{s}^{-1} \mathrm{~cm}^{-2} \AA^{-1}\end{array}$ & $\begin{array}{c}F(2-10 \mathrm{keV}) \\
10^{-11} \mathrm{erg} \mathrm{s}^{-1} \mathrm{~cm}^{-2}\end{array}$ & \begin{tabular}{c}
$\alpha_{\mathrm{UV}-\mathrm{X}}$ \\
\hline 674.5
\end{tabular} \\
\hline \hline 22.56 & $2.79 \pm 0.40$ & $-1.372 \pm 0.051$ \\
1009.5 & 23.27 & $2.52 \pm 0.20$ & $-1.393 \pm 0.028$ \\
1070.5 & 22.56 & $2.95 \pm 0.24$ & $-1.364 \pm 0.029$ \\
2621.5 & 3.78 & $1.58 \pm 0.03$ & $-1.185 \pm 0.007$ \\
2994.5 & 1.31 & $0.74 \pm 0.03$ & $-1.139 \pm 0.014$ \\
4851 & 4.61 & 3.09 & -1.113 \\
5219 & 6.78 & 2.98 & -1.178 \\
\hline \hline
\end{tabular}

NOTE: The first five data are taken from Morini et al. (1986) and the last two ones from Makino (private communication).

\subsection{Variability characteristics}

\subsubsection{The ultraviolet}

The $F(1400 \AA)$ light curve (Fig. 1b) shows a wide range of variability over the duration of the IUE observations. It shows a striking large and relatively slow flux decrease since the first observations (1978-August-02, JD = 723) through 1984 October $29(\mathrm{JD}=3003)$. After this decline, the source brightened again until 1987 June (JD = 3964), where it reached $\approx 1 / 3$ of its maximum brightness. The maximum amplitude of the whole light curve, expressed as the ratio of the maximum to the minimum flux $(R)$, is $R_{\max }=33 \pm 4$ (in order to reduce the formal error on $R_{\max }$ the minimum flux and its error were taken to be the average of the three lowest values and their rms).

Pure Fourier techniques can not be used with unequally sampled data. In this case other algorithms can be applied to allow for the non-uniform sampling. We applied the algorithm by Press \& Teukolsky (1988) to the UV light curve to obtain the frequency power spectrum. This is shown in Fig. 2a, where different significance levels $(0.05,0.001)$ for a random noise hypothesis are also indicated. Small values of this level indicate a highly significant periodic signal. In the figure three main maxima can be observed, corresponding to typical variability time scales of 4903, 182 and 56 days, respectively. The strongest peak at 4903 days is associated with the total duration of the sampling, and does not represent a real variability time scale. Since the mean interval between two consecutive observations is 96 days, we can only expect to resolve variations on longer time scales than this. On the other hand the irregular nature of the sampling could still allow the detection of faster variations. This could indicate the existence of the 56 days peak as a real variability time scale, but since it can not be confirmed from the present data, we conclude that 182 days represents the dominant typical variability time scale of F-9 at $1400 \AA$.

Both $F(1171 \AA)$ and $F(1910 \AA)$ are strongly correlated with $F(1400 \AA)$ and the linear correlation analysis (Figs. 3a, b) gives:

$$
\begin{aligned}
& F_{1171 \AA} \AA=(1.59 \pm 0.10) \times F_{1400 \AA}+ \\
& +(-1.55 \pm 0.65)\left(10^{-14} \mathrm{erg} \mathrm{s}^{-1} \mathrm{~cm}^{-2} \AA^{-1}\right)
\end{aligned}
$$

with a correlation coefficient $r=0.967$; and

$$
\begin{aligned}
& F_{1910 \AA}=(0.65 \pm 0.02) \times F_{1400 \AA} \AA^{+} \\
& +(0.24 \pm 0.15)\left(10^{-14} \mathrm{erg} \mathrm{s}^{-1} \mathrm{~cm}^{-2} \AA^{-1}\right)
\end{aligned}
$$

with $r=0.986$. In both cases, the constant term is small compared to the flux and not significantly different from zero. Most likely the slight deviation from zero of the constant term in relation (6) is caused by the non-linearity and poorly determined calibrations in this region of the IUE camera in the window below $1214 \AA$, while in relation (7) it is due to the small FeII and Balmer continuum contribution to the flux in the $1910 \AA$ window. The strict proportionality of the fluxes in these windows indicates that, in spite of the large amplitude, the brightness variation takes place with constant spectral index between $1171-1910 \AA$. The relations (6) and (7) give spectral indexes of $\alpha(1171-1400 \AA)=0.60 \pm 0.81, \alpha(1400-$ $1910 \AA)=-0.59 \pm 0.23$ and $\alpha(1171-1910 \AA)=-0.17 \pm$ 0.35 . These two last spectral indexes $(\alpha(1400-1910 \AA)$ and $\alpha(1171-1910 \AA))$ are somewhat steeper than the intrinsic UV-optical one $(\approx-0.07$, Sect. 3.1.2). This is most likely caused by the contribution of the Balmer continuum which is correlated with the UV flux (Sect. 4.1) and some FeII contribution (see Figs. 6a-c), resulting in an apparent steepening of the spectral index. Because the Balmer continuum is a nebular component, it is more meaningful to use a large wavelength range to calculate the spectral index linking the UV to the optical, where we have allowed for the removal of non-AGN associated flux in the spectrum, as done in Sect. 3.1.2.

The proportionality between the three UV fluxes and the similarity of their maxima and minima in the light curves, suggests the absence of lag between them. The cross correlation function ( $C C F$; Gaskell \& Peterson 1987) of $F(1171 \AA)$ and $F(1910 \AA)$ with $F(1400 \AA)$ are shown in Fig. 2c and as a reference the auto-correlation function, $A C F$ of $F(1400 \AA)$, in Fig. 2b. These peak at $-4 \pm 67$ and at $0 \pm 65$ days, respectively. This absence of a delay in the variability between the continuum windows, suggest that a coherent emission mechanism is responsible for the continuous emission at all three wavelengths or otherwise the variations are isothermal. 


\subsubsection{The X-rays}

The light curve of the integrated X-ray flux between 2 and $10 \mathrm{keV}$ is clearly different from the UV and optical ones (Fig. 1d). The X-ray light curve decreases from a maximum to a minimum value, after which the flux raises again to its initial level of 1978. The amplitude of variations in this time interval is $R_{\max }=4.0 \pm 0.4$ for the decline and $R_{\max }=4.2 \pm 0.2$ for the increase. This is contrary to the UV continuum, which shows a much larger amplitude of $R_{\mathrm{UV}} \approx 18$ (as calculated from the interpolated values), in the decrease and an amplitude of $R_{\mathrm{UV}} \approx 5$, similar to the $\mathrm{X}$-rays, when brightening. This indicates that both UV and X-rays vary equally in the increase after the minimum, but not in the initial decrease from 1978. This suggests that the good correlation between $\mathrm{X}$-rays and UV breaks down at high levels of the UV continuum (Fig. 4b), as has also been seen in other AGN such as NGC 4151 (Perola et al. 1986) and NGC 5548 (Clavel et al. 1992). Figure $4 \mathrm{~b}$ shows a good correlation whereas the UV flux is weak $\left(<1010^{-14} \mathrm{erg} \mathrm{s}^{-1} \mathrm{~cm}^{-2} \AA^{-1}\right)$, while at UV flux (> $2010^{-14} \mathrm{erg} \mathrm{s}^{-1} \mathrm{~cm}^{-2} \AA^{-1}$ ) the X-rays appear to remain constant. For the intermediate levels, unfortunately, no data are available. This behavior is obviously reproduced in the individual UV-X-rays spectral indexes shown in Table 3. For the low levels the mean spectral index is $\alpha_{\mathrm{UV}-\mathrm{X}}=\alpha(1400 \AA-6 \mathrm{keV})=-1.15 \pm 0.03$, whereas for the high levels it is $\alpha_{\mathrm{UV}-\mathrm{X}}=-1.38 \pm 0.04$, resulting in a significantly steeper spectrum at the high UV brightness, as shown in Fig. 4c.

\subsection{The spectral energy distribution (SED) from radio to $X$-rays}

In Fig. 5 we show the intrinsic F-9 continuum SED (observed), from radio to X-rays, as described above for four different levels of continuum brightness. To allow direct comparison with the standard conventions to display SED in the context of different wavelength domains we show both the frequency spectrum $\log F_{\nu}$ as well as the energy spectrum $\log \nu F_{\nu}$ versus $\log \nu$. The first one allows easy evaluation of the spectral index (slope of the curve) and the second one is the usual way to represent the SED, where a flat SED corresponds to equal energy per decade. In absence of observational support for significant variability in the FIR and radio domain we have used at these wavelengths the single value determined above for all UV and X-ray brightness levels of F-9. For the near IR-hard X-rays range, we use the results of the variability study in each band with respect to the UV one. We will in what follows refer to the four levels of UV brightness as it "high" (JD = 1009.5), "intermediatehigh" (JD = 1951), "intermediate-low" (JD = 2621.5) and "low" (JD = 2994.5), corresponding to the interpolated $F(1400 \AA)$ for each date. We have chosen these dates because for three of them there exist values for the

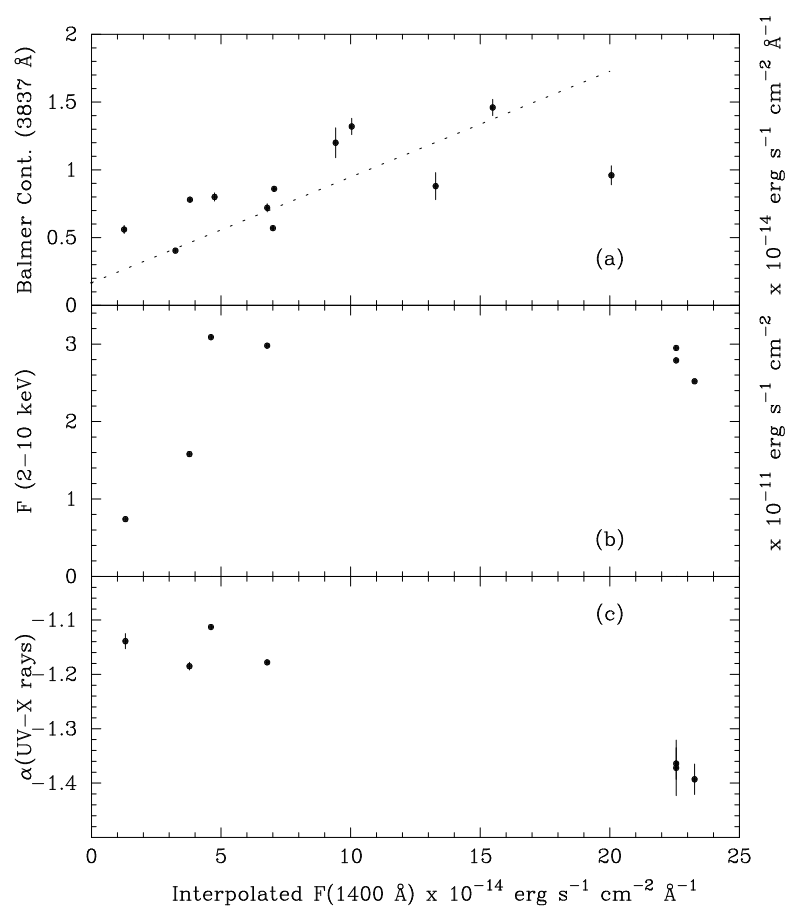

Fig. 4. The Balmer Continuum (BaC), X-rays $(2-10 \mathrm{keV})$ Flux, and the UV to X-rays spectral index $\alpha_{\mathrm{UVX}}$ as a function of the UV continuum brightness $F(1400 \AA)$. a) $\mathrm{BaC}(3837 \AA)$ derived from the data of Lub \& De Ruiter (1992); see Table 2. b) $F(2-10 \mathrm{keV})$; see Table 3 . c) UV-X-rays spectral index $\alpha_{\mathrm{UVX}}$

$F(2-10 \mathrm{keV})$ (see Table 3) and for the JD $=1951$, when there are no X-rays data, we interpolated linearly between the two values nearest to this date, obtaining $F(2-10 \mathrm{keV})=2.1710^{-11} \mathrm{erg} \mathrm{s}^{-1} \mathrm{~cm}^{-2}$. For each date, the $J, H, K$ and $L$ fluxes have been taken by interpolating on the light curves (corrected for the stellar contribution). These observed complete SED's will be applied later, to define the input continuum spectra for the photoionization models in the detailed evaluation of the line intensities and the variability characteristics to disentangle the conditions in the BLR. The flux values used from radio to X-rays, in units of $10^{-14} \mathrm{erg} \mathrm{s}^{-1} \mathrm{~cm}^{-2} \AA^{-1}$ (and between round brackets in units of mJy), are given in Table 4.

From the combined optical, NIR (J) and ultraviolet variability study we found that the spectrum in this domain can be represented, after allowing for the galaxy contribution, by a power law spectrum with a constant spectral index of $\alpha(\mathrm{UV}-$ optical $)=\alpha(\mathrm{UV}-\mathrm{J})=-0.07$ (independently of the brightness). It is then justified to parametrize the central continuum spectrum with a UVoptical-J power law of the type $F_{\nu}=F_{0} \times \nu^{-0.07}$, where $F_{0}$ is determined from the observed $F(1400 \AA)$ for the different brightness levels. In the hard X-rays we use a power law with $\alpha(2-10 \mathrm{keV})=-0.96$ and the constant value is 
Table 4. Flux values used in the SED

$$
\begin{aligned}
& \text { Radio: } F(4.7 \mathrm{GHz})<3.6810^{-11} 10^{-14} \mathrm{erg} \mathrm{s}^{-1} \mathrm{~cm}^{-2} \AA^{-1}(5 \mathrm{mJy}) \\
& \text { Far IR=IRAS bands: } \\
& F(100 \mu \mathrm{m})=0.0013810^{-14} \mathrm{erg} \mathrm{s}^{-1} \mathrm{~cm}^{-2} \AA^{-1}\left(0.4610^{3} \mathrm{mJy}\right) \\
& F(60 \mu \mathrm{m})=0.003910^{-14} \mathrm{erg} \mathrm{s}^{-1} \mathrm{~cm}^{-2} \AA^{-1}\left(0.4710^{3} \mathrm{mJy}\right) \\
& F(25 \mu \mathrm{m})=0.02610^{-14} \mathrm{erg} \mathrm{s}^{-1} \mathrm{~cm}^{-2} \AA^{-1}\left(0.5510^{3} \mathrm{mJy}\right) \\
& F(12 \mu \mathrm{m})=0.07910^{-14} \mathrm{erg} \mathrm{s}^{-1} \mathrm{~cm}^{-2} \AA^{-1}\left(0.3810^{3} \mathrm{mJy}\right) \\
& \text { Near IR-UV-X-rays: } 10^{-14} \mathrm{erg} \mathrm{s}^{-1} \mathrm{~cm}^{-2} \AA^{-1} \text { (mJy) } \\
& \begin{array}{ccccc} 
& \text { HIGH } & \text { INT-HIGH } & \text { INT-LOW } & \text { LOW } \\
F(L=3.35 \mu \mathrm{m})= & 0.33(123.89) & 0.35(132.41) & 0.26(97.82) & 0.18(65.75) \\
F(K=2.10 \mu \mathrm{m})= & 0.50(73.79) & 0.46(67.93) & 0.28(40.78) & 0.16(23.37) \\
F(H=1.58 \mu \mathrm{m})= & 0.47(39.26) & 0.37(31.11) & 0.17(14.46) & 0.09(7.14) \\
F(1400 \AA)= & 23.27(15.20) & 10.04(6.56) & 3.78(2.47) & 1.31(0.86) \\
F(6 \mathrm{keV})= & 766.14(0.00109) & 659.73(0.00094) & 480.36(0.00068) & 224.98(0.00032)
\end{array}
\end{aligned}
$$

determined from the observed $F(2-10 \mathrm{keV})$. This spectral index is taken from Morini et al. (1986) and is within the errors the same as that independently determined from the GINGA observations (see Sect. 3.1.4). There is only one observation with ROSAT, simultaneous with GINGA and IUE. Since the wavelength ranges around the soft Xrays are variable, the soft $\mathrm{X}$-rays are likely to vary also. We can then not use this unique datum for the four brightness levels defined before (Fig. 5). However, the simultaneity with the hard X-rays and the UV on JD $=5219$, allows us to characterize at least part of the big blue bump shape (Fig. 5c). In this figure we have used the ROSAT-GINGA spectral model by Walter (see Sect. 3.1.4), and the J-UV power law with $\alpha=-0.07$ fitted to the $F(1400 \AA)$ in this date. A strong soft X-ray excess is clearly seen with respect to the hard X-ray power law, suggesting that the soft X-rays merge smoothly into the decrease of the big blue bump, which must than have its maximum at energies less than $0.15 \mathrm{keV}(=11 \mathrm{ryd} ; \log \nu=16.6 \mathrm{~Hz})$.

\section{Analysis of the emission lines}

The comparison of the photoionization models with the observations will of course also require a detailed analysis of the emission lines over the large range of variability displayed by F-9. Especially the large amplitude of the variability offers a good opportunity to evaluate the possible existence of different response characteristics over the extent of the broad lines. The line analysis has been done in three stages following the same procedures as applied by Wamsteker et al. (1990) for NGC 5548: first we have removed the various continuum contributions, since especially the small blue bump, mainly composed of Balmer continuum and the many blended FeII lines, form a semicontinuum, which could affect the final results of the line analysis. After the continuum has been properly allowed
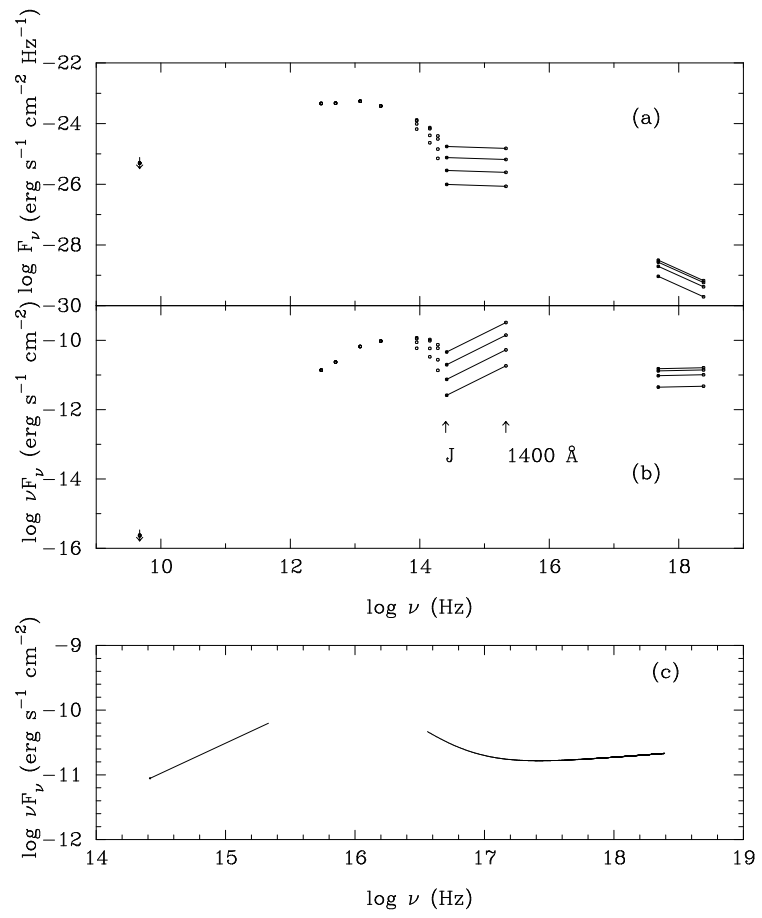

Fig. 5. Different representations of the SED of the F-9 continuum. a) $\log F_{\nu}$ versus $\log \nu$ from radio to X-rays for four levels of UV brightness (see Sect. 3.3). b) $\log \nu F_{\nu}$ versus $\log \nu$ from radio to X-rays for four levels of UV brightness. c) SED $\left(\log \nu F_{\nu}\right.$ versus $\left.\log \nu\right)$ for the unique simultaneous observation IUE-ROSAT-GINGA (JD = 5219). The extrapolation of the hard X-rays $(\log \nu \geq 17.7 \mathrm{~Hz})$ into the soft X-rays clearly indicates a soft $\mathrm{X}$-rays excess. This suggest that the turnover of the big blue bump, which starts at $\log \nu=14.4 \mathrm{~Hz}$, occurs in this range 
for, we are left with a pure emission line spectrum in which one can reliably measure the integrated line intensities. The third part is the detailed line decomposition from essentially pure emission line spectra, and derive the details of the line response for the different velocity domains over the broad line profile. In this last part the actual line variability has been used through the differencing method to derive a line model which will allow the component decomposition for all lines in an internally consistent way for all brightness levels with a minimum number of components.

\subsection{Isolating the lines: The power law continuum, the Balmer continuum and the UV FeII lines}

The nuclear continuum emission, as derived above in Sect. 3.1.2, has been applied to the UV spectra, i.e. a variable power law spectrum, scaled to $F(1400 \AA)$, with constant spectral index $\alpha=-0.07 \pm 0.02$ has been subtracted from each spectrum. The Balmer continuum $(\mathrm{BaC})$ and the UV FeII semi-continuum have been allowed for using the synthetic models by Wills et al. (1985) as applied by Wamsteker et al. (1985) to the optical, in the region of $\mathrm{H} \beta \lambda 4861$, with a gaussian velocity smoothing of $4000 \mathrm{~km} \mathrm{~s}^{-1}$. Although no physical conclusions can be derived from the specific model chosen, the $\mathrm{BaC}$ model is characterized by an effective temperature of $T=1.710^{4} \mathrm{~K}$, and an effective optical depth in the Balmer limit of $\tau(3646 \AA)=4$. The FeII models used are characterized by a turbulent velocity of $v_{\mathrm{t}}=3$ $\mathrm{km} \mathrm{s}^{-1}$, and an optical depth in the UV3 multiplet at $2343 \AA$ of $\tau(2343 \AA)=320$. With these theoretical models a fit to the observed semi-continuum is made between $\approx 1500-3800 \AA$ (i.e. the Small Blue Bump), allowing to isolate the broad lines and to measure their total intensity for each spectrum with some reliability. To establish the intensity of the $\mathrm{BaC}$ we used the photometry of Lub \& De Ruiter (1992) as an intermediate step. After subtracting the stellar contribution and the power law continuum we matched the BaC shape using the photometry in the $L, U$, and $W$ bands at respectively $3837 \AA, 3623 \AA$ and $3235 \AA$. At the redshift of F-9, the $L$ band falls at a rest wavelength of $3668 \AA$ (3562-3774 $\AA$ ), essentially free of FeII and centered on the Balmer limit at $3646 \AA$. Therefore, we have used the measured flux at this band to determine the intensity of the $\mathrm{BaC}$. Subtracting those contributions also from the other two bands, we have fitted the intensity to the observed one at $3837 \AA$. From this we choose from the 12 synthetic models the model which gave the minimum residual flux in the $U$ and $W$ bands, because FeII emission in these bands is minimum. To determine the BaC intensity to all UV spectra we used the relation found between the $\mathrm{BC}(3837 \AA)$ and the interpolated $F(1400 \AA)$ (Table 2 and Fig. 4a):

$$
\begin{aligned}
& \mathrm{BaC}(3837 \AA)=(0.078 \pm 0.002) \times F_{1400 \AA} \AA^{+} \\
& +(0.167 \pm 0.006)\left(10^{-14} \mathrm{erg} \mathrm{s}^{-1} \mathrm{~cm}^{-2} \AA^{-1}\right)
\end{aligned}
$$

with $r=0.76$.

This procedure to determine the $\mathrm{BaC}$ from the observations worked very well except when the UV flux was high, larger than $13-1510^{-14} \mathrm{erg} \mathrm{s}^{-1} \mathrm{~cm}^{-2} \AA^{-1}$. Above this brightness it was necessary to decrease the model intensity, because it would rise above the observations. This suggests that the correlation between $\mathrm{BaC}$ and $F(1400 \AA)$ (Eq. 8) does not persist for UV high levels (see also Fig. 4a, where the three last points $(F(1400 \AA)>13$ $10^{-14} \mathrm{erg} \mathrm{s}^{-1} \mathrm{~cm}^{-2} \AA^{-1}$ ) are somewhat indeterminate in this respect). Such dependence of the $\mathrm{BaC}$ intensity with the UV brightness is similar to that shown by the hard X-rays (Figs. 4a, b). After subtracting the $\mathrm{BaC}$ we fit the selected optimum FeII spectrum to the region between CIII] $\lambda 1909$ and $\operatorname{MgII} \lambda 2798$, adjusting its intensity to each observed spectrum. After subtracting these continuum components a pure emission line spectrum remains, where the continuum slope is not any longer a free parameter in the line fitting. This process to isolate the emission lines is illustrated in Figs. 6a-c for three different levels of the UV brightness. When the UV flux is high (Fig. 6a) the $\mathrm{BaC}$ models matched using Eq. (8) are shown too. It is clear that the observed $2000-3200 \AA$ intensity becomes too large. The $\mathrm{BaC}$ and the FeII (UV) total intensities are given in Table 6 and their relation with the UV continuum is shown in Fig. 8. The errors in the $\mathrm{BaC}$ intensity have been calculated from the linear relation (8) and the UV continuum errors. We find a mean relative error of $13 \%$, similar to the error of $8 \%$ obtained for those epochs when more than one spectrum is available. For the FeII uncertainty this second method has been used and a mean relative error of $17 \%$ was assumed for the case when only one observation was available.

\subsection{Line variability: Total intensity}

Our main purpose with respect to the lines is the study of the line variability over the profile structure. Some UV lines, such as CIII] $\lambda 1909$ and HeII $\lambda 1640$, can not be easily analyzed in this way due to low S/N. Since there are still only very few quantitative results available addressing the detailed broad line structure (NGC 3783, Pelat et al. 1981; 3C 390.3, Zheng et al. 1991; NGC 5548, Wamsteker et al. 1990; Fairall-9, Wamsteker et al. 1985), we also report here the total broad line intensities in Tables 5 and 6 . For the lines which have been decomposed, as is the case for Ly $\alpha \lambda 1216$, NV $\lambda 1240$, SiIV $\lambda 1397$, CIV $\lambda 1549$ and $\operatorname{MgII} \lambda 2798$, we have used the results of the next section and give in Table 5 the sum of the intensities of the different components. This allows us furthermore to measure strongly blended lines such as Ly $\alpha \lambda 1216$ and NV $\lambda 1240$. We have used the error measure of Rodríguez-Pascual (1989) and Wamsteker et al. (1990). This method assumes that the component errors are perfectly correlated, and thus gives an upper limit for the error. The relations between the UV continuum and the total line intensities are 
Table 5. Total intensities of the UV lines (1)

\begin{tabular}{|c|c|c|c|c|c|c|}
\hline $\begin{array}{c}\text { JD } \\
-2443000 \\
\end{array}$ & $\begin{array}{c}F_{1400} \underset{\AA}{\AA} \\
10^{-14} \mathrm{erg} \mathrm{s}^{-1} \mathrm{~cm}^{-2} \AA^{-1}\end{array}$ & \multicolumn{5}{|c|}{$10^{-14} \mathrm{erg} \mathrm{s}^{-1} \mathrm{~cm}^{-2}$} \\
\hline 677 & $19.47 \pm 1.55$ & $2233 \pm 186$ & $298 \pm 163$ & $377 \pm 158$ & $9 \overline{956 \pm 184}$ & $\overline{171 \pm 88}$ \\
\hline 723 & $29.85 \pm 1.98$ & $2819 \pm 187$ & $411 \pm 148$ & $640 \pm 159$ & $1003 \pm 212$ & $208 \pm 87$ \\
\hline 891 & $23.92 \pm 1.43$ & $2999 \pm 228$ & $402 \pm 231$ & $505 \pm 155$ & $1025 \pm 212$ & $169 \pm 108$ \\
\hline 956 & $21.79 \pm 2.00$ & $3199 \pm 158$ & $408 \pm 224$ & $561 \pm 180$ & $925 \pm 232$ & \\
\hline 1414 & $16.68 \pm 3.38$ & $2771 \pm 251$ & $329 \pm 293$ & …......... & $1048 \pm 423$ & $110 \pm 173$ \\
\hline 1448 & $20.12 \pm 1.68$ & $2569 \pm 88$ & $256 \pm 242$ & $140 \pm 241$ & $1090 \pm 234$ & $123 \pm 132$ \\
\hline 1605 & $10.03 \pm 0.98$ & $2269 \pm 138$ & $351 \pm 154$ & $362 \pm 144$ & $892 \pm 234$ & $230 \pm 116$ \\
\hline 1694 & $11.76 \pm 1.51$ & $2081 \pm 131$ & $193 \pm 158$ & & $886 \pm 184$ & \\
\hline 1757 & $11.82 \pm 0.84$ & $2345 \pm 96$ & $234 \pm 161$ & $300 \pm 98$ & $912 \pm 133$ & $174 \pm 53$ \\
\hline 1947 & $10.76 \pm 1.45$ & $2221 \pm 98$ & $233 \pm 145$ & $228 \pm 106$ & $842 \pm 139$ & \\
\hline 1957 & $9.84 \pm 1.10$ & $2211 \pm 115$ & $225 \pm 172$ & $262 \pm 112$ & $809 \pm 212$ & $177 \pm 174$ \\
\hline 1966 & $9.58 \pm 1.43$ & $1860 \pm 182$ & $194 \pm 200$ & $127 \pm 127$ & $702 \pm 166$ & $239 \pm 91$ \\
\hline 2022 & $10.60 \pm 1.19$ & $2322 \pm 69$ & $249 \pm 199$ & $200 \pm 114$ & $829 \pm 146$ & $217 \pm 138$ \\
\hline 2026 & $9.51 \pm 1.45$ & $2144 \pm 138$ & $286 \pm 98$ & $350 \pm 190$ & $865 \pm 193$ & \\
\hline 2247 & $7.14 \pm 1.45$ & $1849 \pm 114$ & $137 \pm 122$ & $142 \pm 156$ & $769 \pm 218$ & $131 \pm 264$ \\
\hline 2251 & $6.00 \pm 1.13$ & $1951 \pm 119$ & $256 \pm 130$ & $225 \pm 133$ & $779 \pm 167$ & \\
\hline 2266 & $7.76 \pm 1.52$ & $1784 \pm 230$ & $146 \pm 35$ & $172 \pm 317$ & $875 \pm 272$ & \\
\hline 2282 & $7.99 \pm 0.35$ & $1893 \pm 77$ & $305 \pm 122$ & $246 \pm 98$ & $856 \pm 106$ & $176 \pm 73$ \\
\hline 2618 & $2.90 \pm 0.51$ & $960 \pm 41$ & $121 \pm 99$ & $85 \pm 75$ & $569 \pm 94$ & $182 \pm 34$ \\
\hline 2622 & $3.46 \pm 0.69$ & $969 \pm 32$ & $99 \pm 76$ & $59 \pm 76$ & $544 \pm 70$ & $165 \pm 32$ \\
\hline 2819 & $1.47 \pm 1.83$ & $924 \pm 157$ & $49 \pm 78$ & $159 \pm 216$ & $312 \pm 257$ & \\
\hline 2943 & $1.11 \pm 0.85$ & $591 \pm 96$ & $25 \pm 12$ & ……........ & $191 \pm 130$ & $139 \pm 20$ \\
\hline 2991 & $1.13 \pm 1.13$ & $287 \pm 60$ & $19 \pm 13$ & ………... & $158 \pm 77$ & \\
\hline 2995 & $0.97 \pm 0.56$ & $333 \pm 48$ & $41 \pm 6$ & ............... & $163 \pm 59$ & $64 \pm 55$ \\
\hline 2999 & $1.62 \pm 0.45$ & $429 \pm 64$ & $30 \pm 12$ & & $253 \pm 65$ & $125 \pm 18$ \\
\hline 3003 & $0.85 \pm 0.54$ & $425 \pm 29$ & $53 \pm 49$ & $73 \pm 52$ & $148 \pm 76$ & \\
\hline 3272 & $3.43 \pm 0.46$ & $754 \pm 64$ & $104 \pm 56$ & $117 \pm 56$ & $330 \pm 77$ & $174 \pm 28$ \\
\hline 3426 & $2.52 \pm 0.62$ & $974 \pm 53$ & $120 \pm 69$ & $134 \pm 79$ & $431 \pm 103$ & $210 \pm 32$ \\
\hline 3533 & $5.78 \pm 0.52$ & $953 \pm 85$ & $121 \pm 55$ & $101 \pm 94$ & $515 \pm 121$ & $154 \pm 27$ \\
\hline 3542 & $6.97 \pm 1.13$ & $941 \pm 73$ & $99 \pm 106$ & $134 \pm 85$ & $464 \pm 110$ & $109 \pm 36$ \\
\hline 3564 & $8.24 \pm 0.73$ & $1138 \pm 90$ & $151 \pm 114$ & $173 \pm 132$ & $473 \pm 104$ & $77 \pm 53$ \\
\hline 3654 & $4.19 \pm 0.47$ & $1080 \pm 53$ & $134 \pm 80$ & $140 \pm 58$ & $486 \pm 83$ & \\
\hline 3729 & $6.38 \pm 0.65$ & $1113 \pm 110$ & $172 \pm 77$ & $107 \pm 119$ & $497 \pm 144$ & $144 \pm 49$ \\
\hline 3731 & $6.55 \pm 0.69$ & $1133 \pm 73$ & $180 \pm 108$ & $137 \pm 99$ & $489 \pm 140$ & $143 \pm 49$ \\
\hline 3794 & $5.31 \pm 0.80$ & $1047 \pm 83$ & $150 \pm 70$ & $133 \pm 100$ & $522 \pm 117$ & $159 \pm 43$ \\
\hline 3820 & $8.07 \pm 0.62$ & $1229 \pm 114$ & $197 \pm 94$ & $127 \pm 141$ & $557 \pm 131$ & $152 \pm 41$ \\
\hline 3924 & $8.38 \pm 1.31$ & $1256 \pm 83$ & $154 \pm 105$ & $158 \pm 105$ & $559 \pm 142$ & $171 \pm 43$ \\
\hline 3964 & $10.70 \pm 1.06$ & $1263 \pm 86$ & $160 \pm 119$ & $107 \pm 150$ & $521 \pm 149$ & $138 \pm 32$ \\
\hline 4020 & $8.86 \pm 1.00$ & $1458 \pm 144$ & $238 \pm 82$ & $213 \pm 159$ & $440 \pm 106$ & $125 \pm 41$ \\
\hline 4381 & $7.64 \pm 0.77$ & $1470 \pm 100$ & $203 \pm 92$ & $165 \pm 128$ & $489 \pm 126$ & $125 \pm 36$ \\
\hline 4448 & $7.82 \pm 0.76$ & $1446 \pm 119$ & $215 \pm 117$ & $239 \pm 151$ & $699 \pm 121$ & $153 \pm 48$ \\
\hline 4528 & $9.97 \pm 0.88$ & $1135 \pm 131$ & $172 \pm 76$ & $129 \pm 115$ & $508 \pm 131$ & $142 \pm 44$ \\
\hline 4656 & $3.71 \pm 0.47$ & $1089 \pm 71$ & $183 \pm 85$ & $100 \pm 80$ & $587 \pm 83$ & $165 \pm 50$ \\
\hline 4729 & $6.33 \pm 0.85$ & $1129 \pm 79$ & $142 \pm 93$ & $75 \pm 95$ & $587 \pm 121$ & $148 \pm 27$ \\
\hline 5117 & $8.31 \pm 0.93$ & $1538 \pm 60$ & $164 \pm 116$ & $229 \pm 112$ & $648 \pm 131$ & $129 \pm 44$ \\
\hline 5219 & $4.48 \pm 0.75$ & $1263 \pm 146$ & $201 \pm 168$ & $204 \pm 121$ & $580 \pm 171$ & $151 \pm 35$ \\
\hline 5268 & $8.72 \pm 0.86$ & $1356 \pm 102$ & $188 \pm 116$ & $219 \pm 87$ & $633 \pm 140$ & $122 \pm 39$ \\
\hline 5331 & $6.68 \pm 1.18$ & $1239 \pm 102$ & $187 \pm 108$ & $120 \pm 108$ & $629 \pm 117$ & $126 \pm 30$ \\
\hline 5540 & $5.59 \pm 0.93$ & $1025 \pm 57$ & $144 \pm 100$ & $95 \pm 96$ & $451 \pm 139$ & $142 \pm 32$ \\
\hline 5553 & $6.16 \pm 0.55$ & $1210 \pm 48$ & $191 \pm 85$ & $164 \pm 68$ & $563 \pm 79$ & $116 \pm 32$ \\
\hline 5568 & $6.21 \pm 0.98$ & $1128 \pm 70$ & $129 \pm 98$ & $82 \pm 131$ & $609 \pm 121$ & \\
\hline 5580 & $6.48 \pm 1.03$ & $1239 \pm 52$ & $136 \pm 88$ & $199 \pm 83$ & $684 \pm 113$ & $135 \pm 43$ \\
\hline
\end{tabular}

shown in Figs. 7 and 8. For HeII $\lambda 1640$ and CIII $] \lambda 1909$, we have measured the intensity by direct integration over the line width and the errors correspond to the product of the integration interval with the rms error of the UV continuum (because the continuum determination is the major source of error).

The relations shown in Figs. 7 and 8 display some interesting characteristics:

1. Most high ionization lines (Ly $\alpha \lambda 1216, \operatorname{NV} \lambda 1240$, SiIV $\lambda 1397, \operatorname{CIV} \lambda 1549, \operatorname{HeII} \lambda 1640$ and CIII] $\lambda 1909)$ are strongly correlated with the continuum, increasing in intensity with the continuum. The simultaneous variation of the continuum with these many lines of different excitation potentials confirms that photoionization, by the UV continuum between 13.6 and $77.5 \mathrm{eV}$, is the main ionization mechanism and that the continuum at $1400 \AA$ is a direct extension of it. For CIV $\lambda 1549$ this relation breaks down at large continuum brightness (the Wamsteker-Colina effect; Shields et al. 1995). When the continuum level is high (> $1610^{-14} \mathrm{erg} \mathrm{s}^{-1} \mathrm{~cm}^{-2} \AA^{-1}$ ), CIV $\lambda 1549$ does not increase any further. Wamsteker \& Colina (1986) suggested this to be caused by the fact that the emission takes place under matter limited conditions, i.e. optically thin conditions, which have been discussed recently in more detail by Shields et al. 
Table 6. Total intensities of the UV lines (2)

\begin{tabular}{|c|c|c|c|c|c|}
\hline $\begin{array}{c}\text { JD } \\
-2443000 \\
\end{array}$ & $\begin{array}{c}F_{1400} \\
10^{-14} \mathrm{erg} \mathrm{s}^{-1} \mathrm{~cm}^{-2} \AA^{-1}\end{array}$ & $\operatorname{HeII} \lambda 1640$ & $\begin{array}{r}\text { CIII] }] 1909 \\
10^{-14} \mathrm{erg} \\
\end{array}$ & $\begin{array}{l}\text { Balmer C. } \\
\mathrm{s}^{-1} \mathrm{~cm}^{-2} \\
\end{array}$ & FeII (UV) \\
\hline 677 & $19.47 \pm 1.55$ & $278 \pm 163$ & $215 \pm 109$ & $3455 \pm 339$ & $1226 \pm 208$ \\
\hline 723 & $29.85 \pm 1.98$ & $565 \pm 208$ & $188 \pm 139$ & $2073 \pm 185$ & $1226 \pm 208$ \\
\hline 891 & $23.92 \pm 1.43$ & $427 \pm 150$ & $280 \pm 100$ & $3801 \pm 308$ & $1471 \pm 250$ \\
\hline 956 & $21.79 \pm 2.00$ & & & & \\
\hline 1414 & $16.68 \pm 3.38$ & $182 \pm 355$ & $170 \pm 237$ & $2419 \pm 510$ & $1153 \pm 172$ \\
\hline 1448 & $20.12 \pm 1.68$ & $165 \pm 176$ & $153 \pm 118$ & $2073 \pm 211$ & $883 \pm 150$ \\
\hline 1605 & $10.03 \pm 0.98$ & $195 \pm 103$ & $145 \pm 69$ & $2505 \pm 271$ & $1717 \pm 292$ \\
\hline 1694 & $11.76 \pm 1.51$ & & & & \\
\hline 1757 & $11.82 \pm 0.84$ & $228 \pm 88$ & $146 \pm 59$ & $2871 \pm 250$ & $785 \pm 49$ \\
\hline 1947 & $10.76 \pm 1.45$ & & & & \\
\hline 1957 & $9.84 \pm 1.10$ & $211 \pm 116$ & $264 \pm 77$ & $2466 \pm 293$ & $1717 \pm 292$ \\
\hline 1966 & $9.58 \pm 1.43$ & $148 \pm 150$ & $277 \pm 100$ & $2411 \pm 362$ & $1717 \pm 292$ \\
\hline 2022 & $10.60 \pm 1.19$ & $152 \pm 125$ & $216 \pm 83$ & $2621 \pm 320$ & $1226 \pm 208$ \\
\hline 2026 & $9.51 \pm 1.45$ & & & & \\
\hline 2247 & $7.14 \pm 1.45$ & $106 \pm 152$ & $195 \pm 102$ & $1907 \pm 355$ & $1471 \pm 250$ \\
\hline 2251 & $6.00 \pm 1.13$ & & & & \\
\hline 2266 & $7.76 \pm 1.52$ & & & & \\
\hline 2282 & $7.99 \pm 0.35$ & $142 \pm 37$ & 25 & $2083 \pm 131$ & $834 \pm 142$ \\
\hline 2618 & $2.90 \pm 0.51$ & $42 \pm 54$ & & $1036 \pm 134$ & $785 \pm 49$ \\
\hline 2622 & $3.46 \pm 0.69$ & $26 \pm 72$ & $178 \pm 48$ & $1149 \pm 176$ & $736 \pm 125$ \\
\hline 2819 & $1.47 \pm 1.83$ & & & & \\
\hline 2943 & $1.11 \pm 0.85$ & $58 \pm 89$ & $44 \pm 60$ & $669 \pm 197$ & $736 \pm 125$ \\
\hline 2991 & $1.13 \pm 1.13$ & & ........ & & \\
\hline 2995 & $0.97 \pm 0.56$ & $23 \pm 59$ & & $637 \pm 136$ & $245 \pm 42$ \\
\hline 2999 & $1.62 \pm 0.45$ & $15 \pm 47$ & $71 \pm 32$ & $774 \pm 115$ & $490 \pm 83$ \\
\hline 3003 & $0.85 \pm 0.54$ & & & & \\
\hline 3272 & $3.43 \pm 0.46$ & $54 \pm 48$ & $57 \pm 32$ & $1144 \pm 129$ & $1153 \pm 172$ \\
\hline 3426 & $2.52 \pm 0.62$ & $93 \pm 65$ & $32 \pm 43$ & $958 \pm 155$ & $1275 \pm 49$ \\
\hline 3533 & $5.78 \pm 0.52$ & $126 \pm 55$ & $62 \pm 36$ & $1629 \pm 152$ & $883 \pm 150$ \\
\hline 3542 & $6.97 \pm 1.13$ & $97 \pm 119$ & $118 \pm 79$ & $1873 \pm 283$ & $736 \pm 125$ \\
\hline 3564 & $8.24 \pm 0.73$ & $106 \pm 77$ & $54 \pm 51$ & $2135 \pm 209$ & $883 \pm 150$ \\
\hline 3654 & $4.19 \pm 0.47$ & & & & \\
\hline 3729 & $6.38 \pm 0.65$ & $222 \pm 68$ & $126 \pm 46$ & $1752 \pm 184$ & $1226 \pm 208$ \\
\hline 3731 & $6.55 \pm 0.69$ & $144 \pm 72$ & $74 \pm 48$ & $1787 \pm 195$ & $1226 \pm 208$ \\
\hline 3794 & $5.31 \pm 0.80$ & $114 \pm 84$ & $95 \pm 56$ & $1532 \pm 208$ & $1349 \pm 71$ \\
\hline 3820 & $8.07 \pm 0.62$ & $172 \pm 65$ & $83 \pm 43$ & $2101 \pm 187$ & $1226 \pm 208$ \\
\hline 3924 & $8.38 \pm 1.31$ & $136 \pm 138$ & $178 \pm 92$ & $2164 \pm 331$ & $736 \pm 125$ \\
\hline 3964 & $10.70 \pm 1.06$ & $182 \pm 111$ & $65 \pm 74$ & $2644 \pm 294$ & $392 \pm 67$ \\
\hline 4020 & $8.86 \pm 1.00$ & $388 \pm 105$ & $220 \pm 70$ & $2261 \pm 267$ & $1226 \pm 208$ \\
\hline 4381 & $7.64 \pm 0.77$ & $186 \pm 81$ & $145 \pm 54$ & $2012 \pm 215$ & $883 \pm 150$ \\
\hline 4448 & $7.82 \pm 0.76$ & $145 \pm 80$ & $199 \pm 53$ & $2052 \pm 213$ & $883 \pm 150$ \\
\hline 4528 & $9.97 \pm 0.88$ & $89 \pm 92$ & $128 \pm 62$ & $1037 \pm 104$ & $490 \pm 83$ \\
\hline 4656 & $3.71 \pm 0.47$ & $64 \pm 49$ & $188 \pm 33$ & $1203 \pm 130$ & $1349 \pm 123$ \\
\hline 4729 & $6.33 \pm 0.85$ & $54 \pm 89$ & $153 \pm 60$ & $1742 \pm 219$ & $932 \pm 294$ \\
\hline 5117 & $8.31 \pm 0.93$ & $186 \pm 98$ & $244 \pm 65$ & $2130 \pm 251$ & $981 \pm 167$ \\
\hline 5219 & $4.48 \pm 0.75$ & $190 \pm 79$ & $158 \pm 53$ & $1362 \pm 195$ & $1471 \pm 250$ \\
\hline 5268 & $8.72 \pm 0.86$ & $217 \pm 90$ & $149 \pm 60$ & $2235 \pm 239$ & $490 \pm 83$ \\
\hline 5331 & $6.68 \pm 1.18$ & $126 \pm 124$ & $259 \pm 83$ & $1815 \pm 294$ & $981 \pm 167$ \\
\hline 5540 & $5.59 \pm 0.93$ & $77 \pm 98$ & $145 \pm 65$ & $1590 \pm 235$ & $766 \pm 41$ \\
\hline 5553 & $6.16 \pm 0.55$ & $129 \pm 58$ & $151 \pm 39$ & $1708 \pm 162$ & $736 \pm 125$ \\
\hline 5568 & $6.21 \pm 0.98$ & & & & \\
\hline 5580 & $6.48 \pm 1.03$ & $128 \pm 108$ & $150 \pm 72$ & $1773 \pm 262$ & $736 \pm 125$ \\
\hline
\end{tabular}

Table 7. Variations amplitudes of the lines and components intensities

\begin{tabular}{|c|c|c|c|c|c|c|c|c|}
\hline \hline$R_{\max }$ & NV & HeII & CIV & SiIV & CIII $]$ & Ly $\alpha$ & MgII & Balmer C. \\
\hline Total int. & $22 \pm 17$ & $40 \pm 120$ & $7 \pm 4$ & $9 \pm 7$ & $9 \pm 12$ & $11 \pm 2$ & - & $6 \pm 1$ \\
\hline Central int. & - & - & $10 \pm 3$ & $9 \pm 5$ & - & $20 \pm 4$ & $4 \pm 1$ & - \\
\hline Red int. & $5 \pm 5$ & - & $7 \pm 6$ & $6 \pm 4$ & - & $19 \pm 7$ & - & - \\
\hline Blue int. & - & - & $6 \pm 3$ & $8 \pm 10$ & - & $14 \pm 11$ & - & - \\
\hline \hline $\begin{array}{c}\text { Ionization } \\
\text { Potential (eV) }\end{array}$ & 77.5 & 54.4 & 48 & 33.5 & 24.4 & 13.6 & 8 & - \\
\hline
\end{tabular}

NOTE: $R_{\max }$ is the ratio between the maximun and minimun intensity. There is no clear dependence between the amplitudes and the lines ionization potential. 


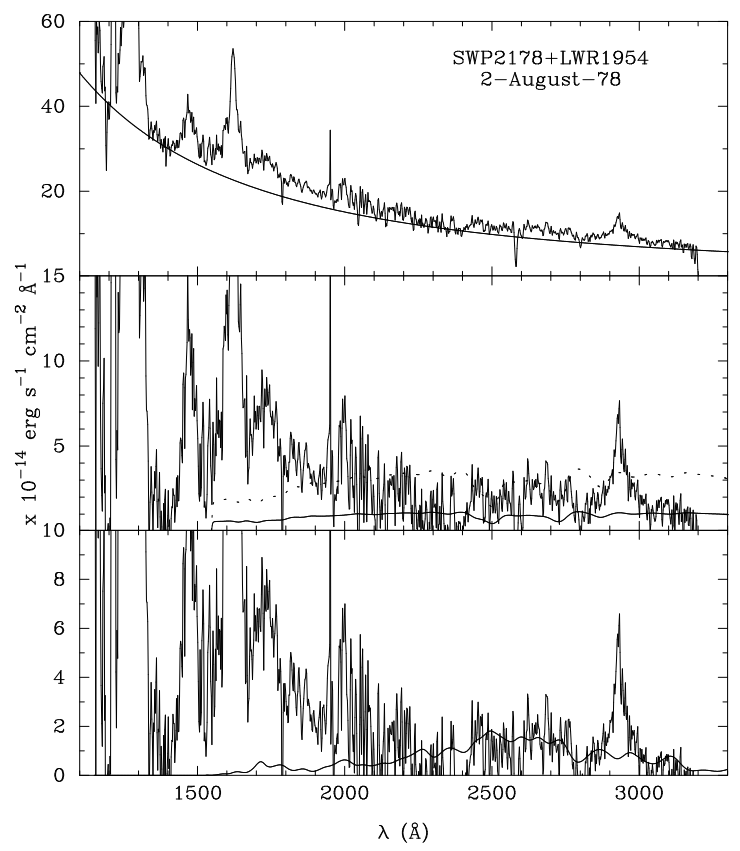

Fig. 6. a) Fit to the UV continuum with a power law, Balmer continuum and the FeII model for a spectrum at high UV level. Upper panel: Observed spectrum with the power-law continuum, corrected for extinction. Middle panel: Spectrum after subtraction of the power law continuum; the Balmer continuum model is also shown. The dotted line shows the model obtained from the application of Eq. (8) at high levels; the actually applied model for the high UV levels is shown as a full drawn line. Bottom panel: Spectrum after subtraction of the power-law continuum and the Balmer continuum, together with the FeII model used (solid line)

(1995). The amplitude of the line intensity variations (Table 7) does not show significant differences between the lines. Clavel et al. (1989) found from the $C C F s$ for Ly $\alpha \lambda 1216$ and CIV $\lambda 1549$ delays of $115 \pm 70$ and $200 \pm 80$ days, respectively, very similar within the errors, suggesting only limited stratification of the BLR. On the other hand the difference in line response characteristics seen in Fig. 7 and the WamstekerColina effect may hide a real stratification. Koratkar \& Gaskell (1989) obtain for CIV $\lambda 1549$ a delay of $124 \pm 39$ days and Lub \& De Ruiter (1992) estimate for Ly $\alpha \lambda 1216$ and CIV $\lambda 1549$ a delay of $220 \pm 140$ days with respect to the optical continuum in the $B$ band $(4298 \AA)$. Attempts to determine the transfer function (TF) through detailed Echo-mapping using both MEMECHO (Horne et al. 1991) as well as the Regularization method (Vio et al. 1994), do indicate the presence of two peaks in the TF for Ly $\alpha \lambda 1216$ : a primary peak at 100 days and a secondary peak at 380 days. No response is indicated at zero delay. These results are, however, rather unstable and could eas-

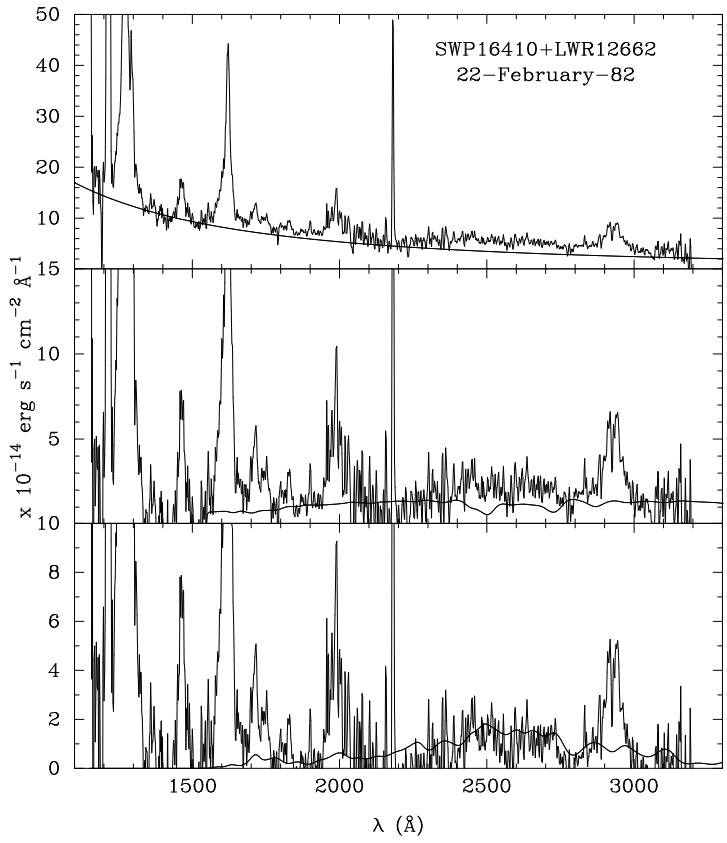

Fig. 6. b) Fit to the UV continuum with a power law, Balmer continuum and the FeII model for a spectrum at intermediate UV level. Upper panel: Observed spectrum with the power-law continuum, corrected for extinction. Middle panel: Spectrum after subtraction of the power law continuum; the Balmer continuum model is also shown. The full drawn line shows the model obtained from the application of Eq. (8). Bottom panel: Spectrum after subtraction of the power-law continuum and the Balmer continuum, together with the FeII model used (solid line)

ily be the consequence of non-linear response of both CIV $\lambda 1549$ and Ly $\alpha \lambda 1216$ to the variations in the ionizing flux. This appears to be confirmed by the fact that the TF for Ly $\alpha \lambda 1216$ and CIV $\lambda 1549$ are essentially mutually exclusive, and no acceptable continuum lightcurve can reproduce the line lightcurves unless the linear response condition is released.

2. The lower ionization emission lines such as $\operatorname{MgII} \lambda 2798$ and FeII show, if any, a weak correlation with the continuum for $F_{1400 \AA}<610^{-14} \mathrm{erg} \mathrm{s}^{-1} \mathrm{~cm}^{-2} \AA^{-1}$. At higher continuum levels they are constant. This can be understood if these lines originate in the inner BLR, where only hard X-rays with energy $>100 \mathrm{eV}$ can penetrate. Although the sampling of the X-ray data is limited, the results shown in Fig. 4b suggest the existence of a similar cutoff in the $F_{1400 \AA}$ vs. $F_{2-10 \mathrm{keV}}$ relation, strongly suggesting that direct $\mathrm{X}$-ray heating is indeed the dominant mechanism for these lines. Clavel et al. (1989) and Koratkar \& Gaskell (1989) found from $C C F$ analysis delays of $160 \pm 90$ days for $\operatorname{MgII} \lambda 2798$ and $167 \pm 61$ days for $\operatorname{Ly} \alpha \lambda 1216$ and $\operatorname{CIV} \lambda 1549$ with 


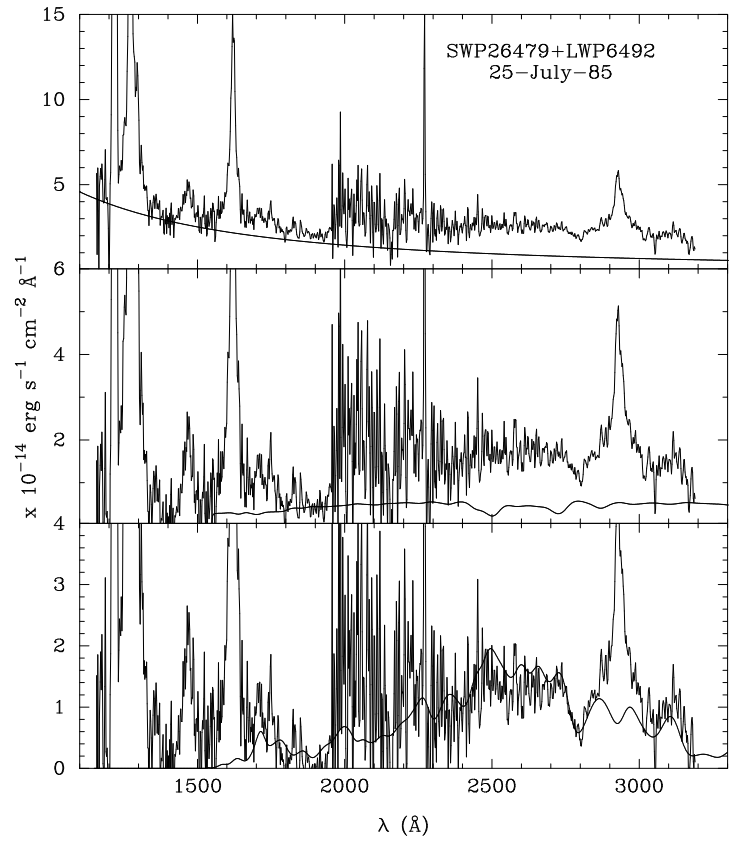

Fig. 6. c) Fit to the UV continuum with a power law, Balmer continuum and the FeII model for a spectrum at low UV level. Upper panel: Observed spectrum with the power-law continuum, corrected for extinction. Middle panel: Spectrum after subtraction of the power law continuum; the Balmer continuum model is also shown. Bottom panel: Spectrum after subtraction of the power-law continuum and the Balmer continuum, together with the FeII model used (solid line)

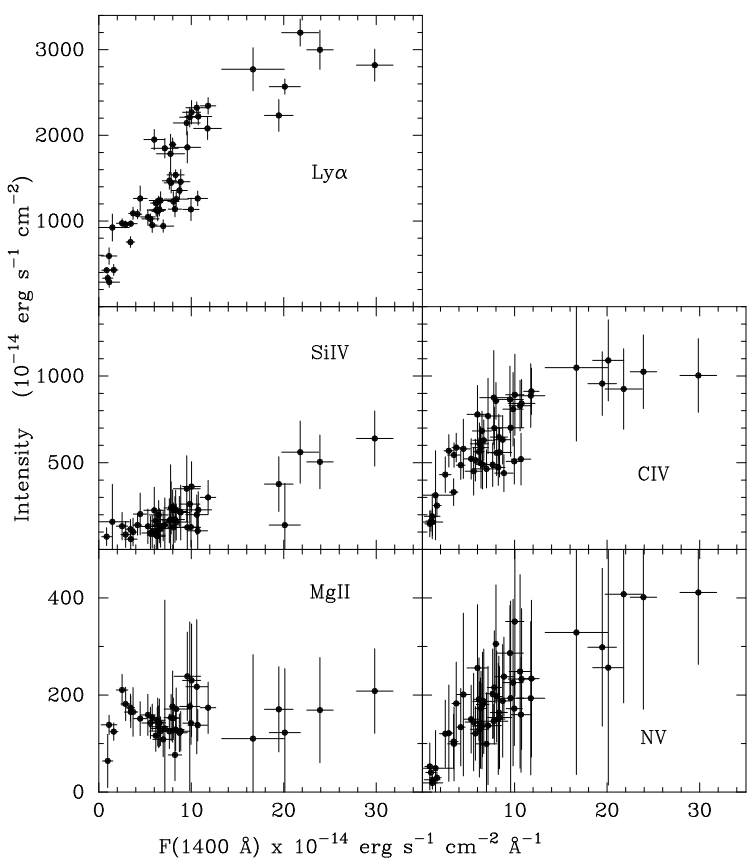

Fig. 7. Total intensities of the UV lines versus $F(1400 \AA)(1)$

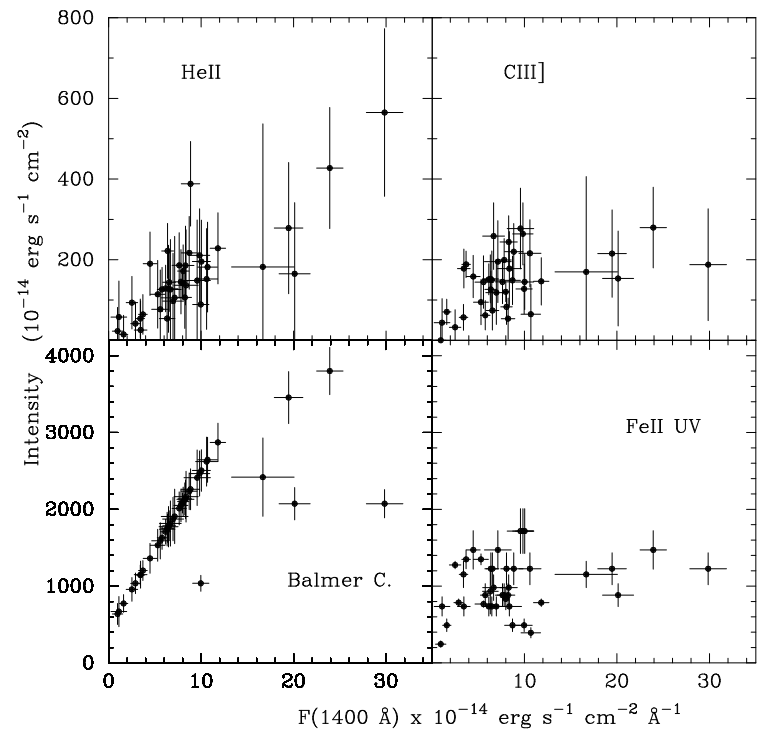

Fig. 8. Total intensities of the UV lines versus $F(1400 \AA)(2)$

respect to the UV continuum. Lub \& De Ruiter (1992) estimate the MgII delay at $330 \pm 140$ days with respect to the optical continuum.

3. The BaC shows a very tight correlation with the UV continuum, which also appears to break down, similarly to CIV $\lambda 1549$, at continuum brightness above $F_{1400 \AA}>1610^{-14} \mathrm{erg} \mathrm{s}^{-1} \mathrm{~cm}^{-2} \AA^{-1}$. This could be understood in similar terms as for the low ionization lines except for the fact that a substancial part of the $\mathrm{BaC}$ emitting region is presumably heated by the lower energy part of the continuum just beyond the Lyman limit. As a consequence, the $\mathrm{BaC}$ can continue to respond to the increase in the UV brightness beyond levels shown by the low ionization lines.

The average line ratios for the strongest lines are given in Table 8. The optical lines ratios have been calculated from the observed intensities by Lub \& De Ruiter (1992), with the UV values interpolated as before, to determine values simultaneous to the optical data. Wills et al. (1985) obtain for their sample of Seyfert 1 galaxies a value of FeII $($ total $=\mathrm{OP}+\mathrm{UV}) / \mathrm{MgII} \lambda 2798 \approx 8$, similar to our result of FeII (UV) $/ \mathrm{MgII}=7 \pm 2$ (in our case the optical multiplets have not been summmed; see Table 8 ). These authors explain this large value by an overabundance of $\mathrm{Fe}$, by a factor of $\approx 3$ with respect to the cosmic abundance.

\subsection{Variability in the line profile}

\subsubsection{Profile decomposition}

We have applied the difference method to this homogeneous set of spectra over a long temporal interval and large variability to isolate the variable components in 
Table 8. Observed lines ratios and comparation with the theoretical prediction by Kwan \& Krolik (1981)

\begin{tabular}{|c|c|c|c|c|c|}
\hline Ratio & $\begin{array}{l}\text { Observed } \\
\text { mean value }\end{array}$ & $\begin{array}{l}\text { theoretical value } \\
\text { (Kwan and Krolik) }\end{array}$ & $\begin{array}{l}\text { Depending } \\
\text { parameters }\end{array}$ & $\begin{array}{c}\text { Implications } \\
\text { of the } \\
\text { observed value }\end{array}$ & Notes \\
\hline "Ly $\alpha \lambda 1216 / \mathrm{NV} \lambda 1240$ & $9 \pm 3$ & $\overline{12.5}$ & $\overline{\bar{U}}$ & $\bar{U} \geq 0.03$ & \\
\hline Ly $\alpha \lambda 1216 / \operatorname{SiIV} \lambda 1397$ & $9 \pm 3$ & $\approx 10$ & $\ldots$ & $\ldots \ldots$ & Good agreement \\
\hline Ly $\alpha \lambda 1216 / \mathrm{HeII} \lambda 1640$ & $12 \pm 7$ & $\approx\left(3 \times 4^{-\alpha}\right)$ & $\begin{array}{c}\alpha \\
\text { (between } \\
13.6 \text { and } \\
300 \mathrm{eV} \text { ) }\end{array}$ & $\alpha \approx-1$ & $\begin{array}{c}\text { Index similar to } \\
\text { the observed one } \\
\alpha_{\mathrm{UV}-\mathrm{X}=-1.15 \text { or }-1.38}=-1 \text { (Sect. } 3.2 .5 \text { ) }\end{array}$ \\
\hline CIII] $\lambda 1909 /$ CIV $\lambda 1549$ & $0.24 \pm 0.09$ & 0.20 & $N_{\mathrm{e}}$ & $N_{\mathrm{e}} \leq 410^{9} \mathrm{~cm}^{-3}$ & $\begin{array}{c}\text { Presence } \\
\text { of CIII }] \Rightarrow \\
N_{\mathrm{e}}<3.9810^{9} \mathrm{~cm}^{-3}\end{array}$ \\
\hline Ly $\alpha \lambda 1216 /$ C.Balmer & $0.8 \pm 0.2$ & 2.3 & $\begin{array}{c}N_{\mathrm{col}} \\
N_{\mathrm{e}} \\
\alpha_{\mathrm{X}} \\
\end{array}$ & $\begin{array}{c}N_{\mathrm{col}}>10^{23} \mathrm{~cm}^{-2} \\
\left(N_{\mathrm{e}}>410^{9} \mathrm{~cm}^{-3}\right) \\
\left(\alpha_{\mathrm{X}}>-0.5\right) \\
\end{array}$ & $\begin{array}{c}\text { Adequate solution } \\
\text { Incomp. CIII] } / \mathrm{CIV} \\
\text { Incomp. } \alpha_{\mathrm{X}}(\mathrm{obs})=-0.96 \\
\end{array}$ \\
\hline FeII UV/MgII $\lambda 2798$ & $7 \pm 2$ & $\begin{array}{c}1.3 \\
\text { taking } \\
\text { FeII }(\mathrm{OP}+\mathrm{UV})\end{array}$ & $\ldots$ & ...... & See text \\
\hline $\operatorname{Ly} \alpha \lambda 1216 / \operatorname{MgII} \lambda 2798$ & $\begin{array}{c}10 \pm 4 \\
\text { vary } \\
\text { between } 3 \text { y } 25\end{array}$ & 6 & $\ldots$ & ...... & ...... \\
\hline Ly $\alpha \lambda 1216 / F e I I ~ U V$ & $\begin{array}{c}1.5 \pm 0.7 \\
\text { vary } \\
\text { between } 0.7 \text { y } 3.2\end{array}$ & $\ldots$ & $\ldots$ & ...... & See text \\
\hline Ly $\alpha \lambda 1216 / \mathrm{CIV} \lambda 1549$ & $\begin{array}{c}2.3 \pm 0.4 \\
\text { vary } \\
\text { between } 1.7 \text { y } 3.5 \\
\end{array}$ & 1.6 & $\begin{array}{c}\alpha_{13.6-300 \mathrm{eV}} \\
U\end{array}$ & $(U<0.03)$ & Incomp. Ly $\alpha / \mathrm{NV}$ \\
\hline$\overline{\mathrm{H} \alpha \lambda 6563 / \mathrm{H} \beta \lambda 4861}$ & $\overline{26 \pm 2}$ & 4.16 & $\overline{N_{\mathrm{e}}}$ & $N_{\mathrm{e}} \leq 410^{9} \mathrm{~cm}^{-3}$ & $\overline{c . .1 .}$ \\
\hline $\mathrm{HeI} \lambda 5876 / \mathrm{H} \beta \lambda 4861$ & $0.35 \pm 0.07$ & 0.09 & $\begin{array}{c}U \\
N_{\mathrm{e}}\end{array}$ & $\begin{array}{c}\bar{U}>0.03 \\
\left(N_{\mathrm{e}}>410^{9} \mathrm{~cm}^{-3}\right)\end{array}$ & $\begin{array}{c}\text { Adequate solution } \\
\text { Incomp. } \mathrm{H} \alpha / \mathrm{H} \beta\end{array}$ \\
\hline FeII $\lambda 4570 / \mathrm{H} \beta \lambda 4861$ & $0.8 \pm 0.4$ & 0.21 & $\begin{array}{c}N_{\text {col }} \\
N_{\mathrm{e}} \\
\alpha_{\mathrm{X}} \\
\end{array}$ & $\begin{array}{c}N_{\mathrm{col}}>10^{23} \mathrm{~cm}^{-2} \\
\left(N_{\mathrm{e}}>410^{9} \mathrm{~cm}^{-3}\right) \\
\left(\alpha_{\mathrm{X}}>-0.5\right)\end{array}$ & $\begin{array}{c}\text { Adequate solution } \\
\text { Incomp. } \mathrm{H} \alpha / \mathrm{H} \beta \\
\text { Incomp. } \alpha_{r m X}(\text { obs })=-0.96\end{array}$ \\
\hline Ly $\alpha \lambda 1216 / \mathrm{H} \beta \lambda 4861$ & $26 \pm 4$ & $\overline{10.4}$ & $\begin{array}{c}\alpha_{\mathrm{X}} \\
N_{\mathrm{col}}\end{array}$ & $\begin{array}{c}\alpha_{\mathrm{X}}<-0.5 \\
\left(N_{\mathrm{col}}<10^{23} \mathrm{~cm}^{-2}\right)\end{array}$ & $\begin{array}{l}\text { Comp. } \alpha_{\mathrm{X}}(\mathrm{obs})=-0.96 \\
\text { Incomp. other ratios }\end{array}$ \\
\hline $\operatorname{MgII} \lambda 2798 / \mathrm{H} \beta \lambda 4861$ & $4 \pm 3$ & 1.75 & $N_{\mathrm{col}}$ & $N_{\mathrm{col}} \geq 10^{23} \mathrm{~cm}^{-2}$ & $\ldots \ldots$ \\
\hline C.Balmer $/ \mathrm{H} \beta \lambda 4861$ & $35 \pm 8$ & 4.56 & $\begin{array}{c}N_{\mathrm{col}} \\
N_{\mathrm{e}} \\
\alpha_{\mathrm{X}}\end{array}$ & $\begin{array}{c}N_{\text {col }}>10^{23} \mathrm{~cm}^{-2} \\
\left(N_{\mathrm{e}}>410^{9} \mathrm{~cm}^{-3}\right) \\
\left(\alpha_{\mathrm{X}}>-0.5\right)\end{array}$ & $\begin{array}{c}\text { Adequate solution } \\
\text { Incomp. other ratios } \\
\text { Incomp. } \alpha_{\mathbf{X}}(\text { obs })=-0.96\end{array}$ \\
\hline
\end{tabular}

NOTE: For the ratios sensitive to several parameters it is enough that some of the marked implications be verified. The rejected implications due to their imcompatibility with other ratios are showed between round brackects.

the emission lines. This method has been first applied by Wamsteker et al. (1990) to the UV and optical spectra of NGC 5548 and has here been used for Ly $\alpha \lambda 1216$, $\operatorname{CIV} \lambda 1549$, SiIV $\lambda 1397$ and MgII $\lambda 2798$. With only four components, all line profiles of F-9 could be described over the whole brightness range. These four basic components are identified in Table 9. From a large set of subtracted spectra we determine the average of the $F W H M$, position (= central wavelength) and the height of each component. These are introduced as input parameters in the total profile fit. Similarly to Wamsteker et al. (1990), we represent the components as gaussians. This procedure makes it also possible to measure some of the highly blended lines by introducing the blends as individual components in an overall line fit. For example the Ly $\alpha \lambda 1216-N V \lambda 1240$ blend must be fitted together in the Ly $\alpha \lambda 1216$ region, to account for the NV $\lambda 1240$ presence (one narrow and one red component). In CIV $\lambda 1549$, a very weak component at $\lambda=1491 \AA$ is most likely associated with NIV] $\lambda 1486$.
The fitting with $\chi^{2}$ minimization (the ESO-IHAP procedures have been used) proceeded in two phases: first, the width and the position are fixed, allowing only the height to vary. After this fit has converged, position and $F W H M$ are released to assure that the solution remains stable in these two parameters as well. To illustrate the results of the gaussian fitting to the data with the line model of Table 9, we show in Fig. 9 the results for Ly $\alpha \lambda 1216$ and CIV $\lambda 1549$ at three different levels of brightness. The broad emission lines show the following components structure: a narrow component with $F W H M \approx 1700 \mathrm{~km} \mathrm{~s}^{-1}$ (unresolved), a central broad component at the same velocity as the narrow line with $F W H M \approx 3600 \mathrm{~km} \mathrm{~s}^{-1}$, a broad red component with $F W H M \approx 5800 \mathrm{~km} \mathrm{~s}^{-1}$ and relative velocity of $\approx 3300 \mathrm{~km} \mathrm{~s}^{-1}$, and a broad blue component with $F W H M \approx 5900 \mathrm{~km} \mathrm{~s}^{-1}$ and a relative velocity of $\approx-3600 \mathrm{~km} \mathrm{~s}^{-1}$. The intensities in the different line components are given in Tables 10, 11, 12 and 13 for Ly $\alpha \lambda 1216$ (with NV $\lambda 1240$ ), SiIV $\lambda 1397, \operatorname{CIV} \lambda 1549$ and MgII $\lambda 2798$, respectively. The errors are calculated as described by 
Table 9. Profiles components of the UV lines

\begin{tabular}{|c|c|c|c|c|c|c|}
\hline Components & Characteristics & Ly $\alpha \lambda 1216$ & NV $\lambda 1240$ & CIV $\lambda 1549$ & SiIV $\lambda 1397$ & MgII $\lambda 2798$ \\
\hline \multirow{2}{*}{ Narrow } & Width (km/s) & $1726 \pm 260$ & $1916 \pm 322$ & $1692 \pm 306$ & $1165 \pm 641$ & $1365 \pm 380$ \\
\hline & Rel. vel. $(\mathrm{km} / \mathrm{s})$ & 0 & 0 & 0 & 0 & 0 \\
\hline \multirow{2}{*}{ Central } & Width $(\mathrm{km} / \mathrm{s})$ & $3672 \pm 384$ & & $3617 \pm 399$ & $3534 \pm 560$ & $3564 \pm 545$ \\
\hline & Rel. vel. $(\mathrm{km} / \mathrm{s})$ & $47 \pm 236$ & & $-18 \pm 278$ & $-266 \pm 1186$ & $174 \pm 389$ \\
\hline \multirow{2}{*}{ Red } & Width $(\mathrm{km} / \mathrm{s})$ & $5905 \pm 442$ & $4534 \pm 965$ & $5766 \pm 1109$ & $6684 \pm 1475$ & $5883 \pm 1354$ \\
\hline & Rel. vel. $(\mathrm{km} / \mathrm{s})$ & $3280 \pm 354$ & $3814 \pm 486$ & $2979 \pm 611$ & $3498 \pm 1821$ & $3146 \pm 738$ \\
\hline \multirow{2}{*}{ Blue } & Width $(\mathrm{km} / \mathrm{s})$ & $6597 \pm 903$ & & $5731 \pm 936$ & $6105 \pm 1304$ & $5306 \pm 846$ \\
\hline & Rel. vel. $(\mathrm{km} / \mathrm{s})$ & $-3940 \pm 590$ & & $-3738 \pm 611$ & $-3641 \pm 1493$ & $-3238 \pm 912$ \\
\hline Others & $\begin{array}{l}\text { Width }(\mathrm{km} / \mathrm{s}) \\
\text { Rel. vel. }(\mathrm{km} / \mathrm{s})\end{array}$ & & & $\begin{array}{c}3561 \pm 903 \\
-11178 \pm 851\end{array}$ & & \\
\hline
\end{tabular}

Rodríguez-Pascual (1989) and Wamsteker et al. (1990) using the errors in the total line intensity. The relative errors in the total intensity are for $\operatorname{Ly} \alpha \lambda 1216, \operatorname{CIV} \lambda 1549$, $\operatorname{MgII} \lambda 2798, \mathrm{NV} \lambda 1240$ and $\operatorname{SiIV} \lambda 1397$ 8\%, 26\%, 44\%, $63 \%$ and $85 \%$, respectively.

\subsubsection{The narrow component}

The width of the narrow lines is defined by the resolution of IUE at a FWHM of $\approx 1700 \mathrm{~km} \mathrm{~s}^{-1}$ (Table 9). The fractional contribution of the narrow line to the total line intensity is at $23,17,11,52$ and $1 \%$ for $\operatorname{Ly} \alpha \lambda 1216$, $\operatorname{CIV} \lambda 1549, \operatorname{MgII} \lambda 2798, \mathrm{NV} \lambda 1240$ and SiIV $\lambda 1397$, respectively. Although the narrow lines do not show a clear relation with $F_{1400 \AA}$, the results do not suggest a pure scatter diagram, but over the time interval of the UV observations no systematic behavior could be identified (see Fig. 10). The average intensity ratios for the different narrow components of the lines are Ly $\alpha \lambda 1216 / \mathrm{NV} \lambda 1240$ $\approx 3, \operatorname{Ly} \alpha \lambda 1216 / \operatorname{SiIV} \lambda 1397 \approx 19, \operatorname{Ly} \alpha \lambda 1216 / \operatorname{CIV} \lambda 1549$ $\approx 3$ and $\operatorname{Ly} \alpha \lambda 1216 / \operatorname{MgII} \lambda 2798 \approx 18$ (with large errors due to the large dispersion in the data).

The line decomposition of $\mathrm{H} \beta \lambda 4861$ by Wamsteker et al. (1985) showed that the intrinsic narrow line width is $670 \mathrm{~km} \mathrm{~s}^{-1}$. Even so the ratio $\operatorname{Ly} \alpha \lambda 1216 / \mathrm{H} \beta \lambda 4861$ is $\approx 69$, two times higher than the Case $\mathrm{B}$ of recombination, but similar to the obtained one by Ferland \& Osterbrock (1986) with their Seyfert 2 galaxies sample $(\operatorname{Ly} \alpha \lambda 1216 / \mathrm{H} \beta \lambda 4861$ (intrinsic) $\approx 55)$. They explain this as due to the fact that the NLR is photo-ionized by a hard optical-X-rays continuum $\left(\alpha_{\mathrm{o}-\mathrm{x}} \approx-1.4\right.$, although also in their data a resolution effect can not be excluded. Our observations (Sect. 3.2.5) also suggest a hard UV-X-rays spectral index, $\alpha_{\mathrm{UV}-\mathrm{x}} \approx-1.15$ or -1.38 .

\subsubsection{The central component}

This component (Table 9) has an average velocity respect to the narrow line of $\approx 0$, while its mean FWHM is $\approx 3600 \mathrm{~km} \mathrm{~s}^{-1}$. Its contribution to the total intensity is respectively $32,38,48$ and $39 \%$ for $\operatorname{Ly} \alpha \lambda 1216$, $\operatorname{CIV} \lambda 1549, \operatorname{MgIII} \lambda 2798$ and $\operatorname{SiIV} \lambda 1397$. Its behavior with respect to the UV continuum (Fig. 10) shows that all the lines correlate with $F_{1400 \AA}$, though CIV $\lambda 1549$ stops increasing at higher levels of continuum $(>10$ $\left.10^{-14} \mathrm{erg} \mathrm{s}^{-1} \mathrm{~cm}^{-2} \AA^{-1}\right)$. As seen in Fig. 11, Ly $\alpha \lambda 1216$ and CIV $\lambda 1549$ appear to show a looping behavior in line vs. continuum flux diagram, strongly suggesting delay with respect to the continuum (in the $\operatorname{Ly} \alpha \lambda 1216$ figure the time sense has been indicated by an arrow). The amplitudes of the central component for the different lines is given in Table 7 .

In Fig. 12 (see also Table 14), we present the $C C F$ (Gaskell \& Peterson 1987) and the Discrete Correlation Function ( $D C F$; Edelson \& Krolik 1988) for the central components with respect to the UV continuum are shown. For the $D C F$ calculus we have chosen an interval between points of 100 days, according to the mean interval between two consecutive observations (96 days). Although with this interval the $D C F$ is noisy, it has the advantage that the time resolution in the data is not degraded. For the $C C F$ we indicate the peak (with its error calculated as Gaskell \& Peterson 1987) and the centroid of the function, calculated as the average of the positive and negative HWHM. The peak of the $C C F$ seems to be more influenced by the response of the gas nearer the source (Robinson \& Pérez 1990; Pérez et al. 1992), while the centroid seems to indicate the radius where the radiation is larger (Koratkar \& Gaskell 1991). For the DCF the delay of the maximum point is given, more compatible with the center of the $C C F$ than with the peak. There are no significant differences between the delays of the $C C F$ centers for $\operatorname{Ly} \alpha \lambda 1216$, CIV $\lambda 1549$, SiIV $\lambda 1397$ and $\operatorname{MgII} \lambda 2798$, so that an averaged delay is $400 \pm 100$ days (average error). This is consistent with the averaged delay derived from the $D C F$ of $450 \pm 175$ days and both values are very 


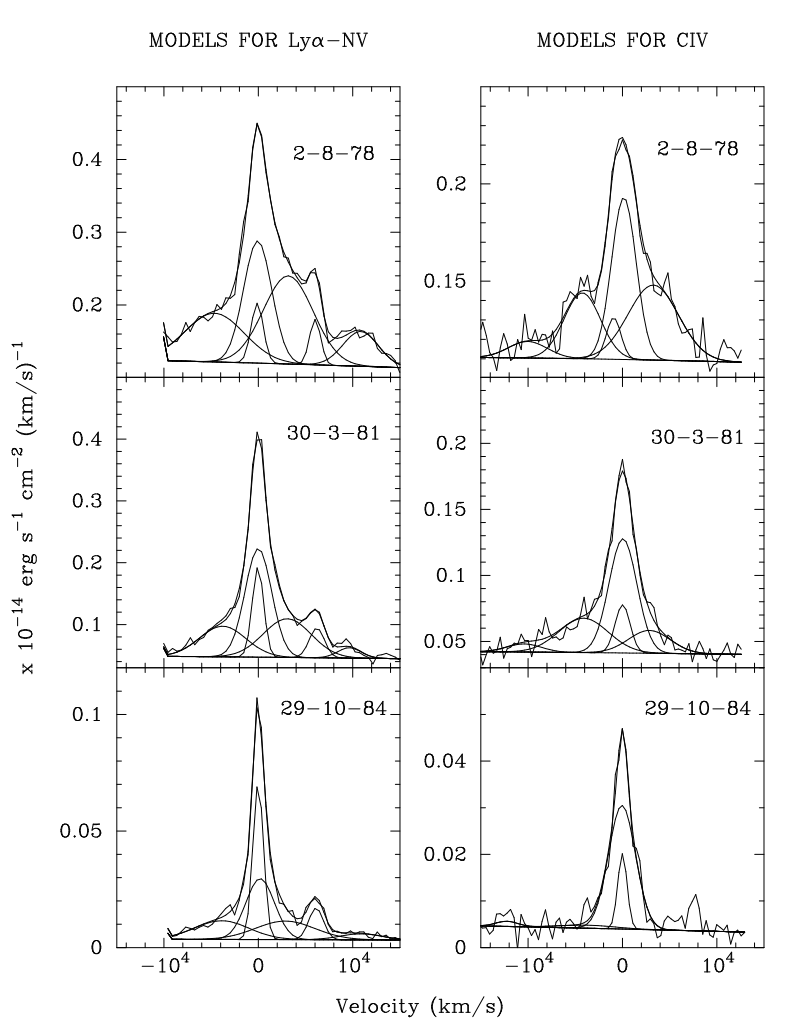

Fig. 9. This figure shows the components and total fit to Ly $\alpha \lambda 1216$ and CIV $\lambda 1549$ for three different brightness levels of the UV continuum (high $=28-8-78$; intermediate $=30-8-81 ;$ and low $=29-10-84$ )

similar to the delay found for the region of hot dust emission of $400 \pm 100$ days by Clavel et al. (1989).

The line ratios for the central components for Ly $\alpha \lambda 1216 / \operatorname{CIV} \lambda 1549$, Ly $\alpha \lambda 1216 / \operatorname{MgIII} \lambda 2798$ as well as Ly $\alpha \lambda 1216 / \operatorname{SiIV} \lambda 1397$ appear to show a slight increase with the UV continuum (Fig. 18). The mean ratios are $2.0 \pm 0.8,8 \pm 4$ and $6 \pm 3$, respectively. This central component is also present in the $\mathrm{H} \beta \lambda 4861$ profile decomposition (Wamsteker et al. 1985), with a $F W H M=3730 \mathrm{~km} \mathrm{~s}^{-1}$ and a relative velocity with respect to the narrow line of $+130 \mathrm{~km} \mathrm{~s}^{-1}$. Interpolating in Ly $\alpha \lambda 1216$ to obtain simultaneous values with the $\mathrm{H} \beta \lambda 4861$ central component a mean ratio Ly $\alpha \lambda 1216 / \mathrm{H} \beta \lambda 4861 \approx 13-15$ is obtained. Lub \& De Ruiter's (1992) optical study do not decompose the Balmer lines, on the other hand they find a strong dependence of the delay versus the $B$ continuum (at $4298 \AA$ ), obtaining $\approx 400$ for $\mathrm{H} \alpha \lambda 6563,270 \pm 150$ for $\mathrm{H} \beta \lambda 4861$ and $100 \pm 120$ days for $\mathrm{H} \gamma \lambda 4340$. Note that this central component could not be discriminated in the lines of HeII $\lambda 1640$ and NV $\lambda 1240$ suggesting that this component is much weaker for these two high ionization lines.

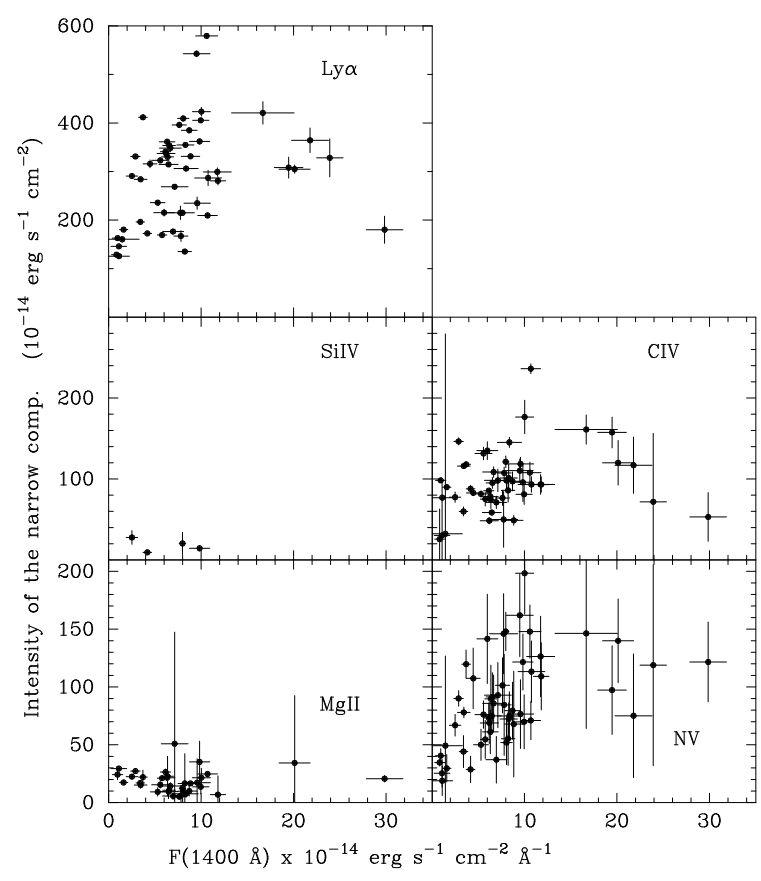

Fig. 10. Intensities of the narrow components of the strong emission lines versus the UV continuum brightness at $1400 \AA$

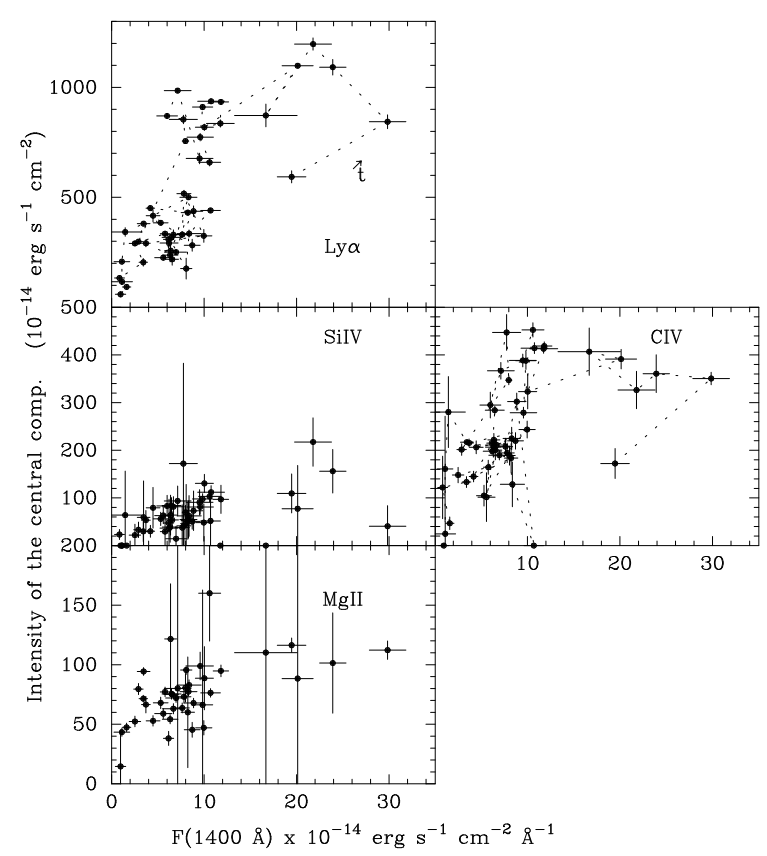

Fig. 11. Intensities of the central components (see Table 9) versus the UV continuum at $1400 \AA$. The arrow in the box for Ly $\alpha \lambda 1216$ (upper left) indicates the direction of time along the points connected by the dotted lines for Ly $\alpha \lambda 1216$ and CIV $\lambda 1549$ 


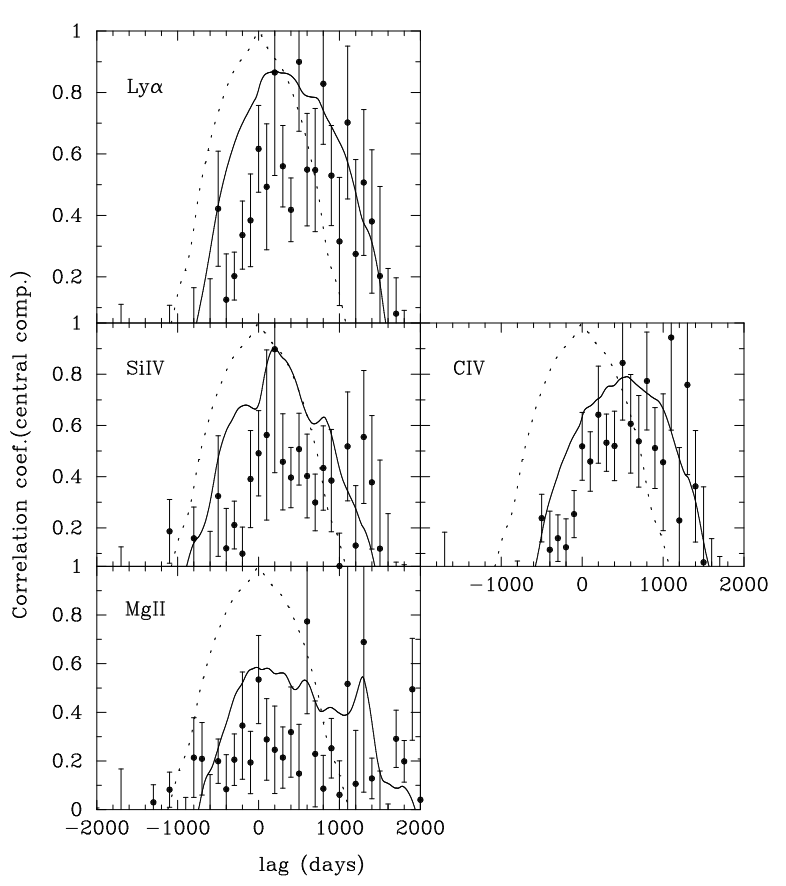

Fig. 12. Correlation functions for the central component. Solid line: Cross Correlation Function (CCF). Points and error bars: Discrete Correlation Function (DCF). Dotted line: Autocorrelation Function (ACF) for the UV continuum at $1400 \AA$

\subsubsection{The red component}

The red component with a $F W H M$ of $\approx 5800 \mathrm{~km} \mathrm{~s}^{-1}$ shows a velocity with respect to the narrow line of $\approx+3300 \mathrm{~km} \mathrm{~s}^{-1}$ (Table 9). It contributes to the total intensity with $26,17,24,48$ and $34 \%$ for Ly $\alpha \lambda 1216$, CIV $\lambda 1549, \operatorname{MgII} \lambda 2798, \operatorname{NV} \lambda 1240$ and SiIV $\lambda 1397$, respectively, somewhat less than the fractional contribution of the central component. The intensity of the red component is very tightly correlated with the UV continuum (Fig. 13) for most of the lines, except MgII $\lambda 2798$, which does not appear to respond to the UV continuum. At the time resolution in our data (96 days), no indication of delay is suggested for any of the high excitation lines. The amplitude of the variations (Table 7 ) are the same within the errors for all high excitation lines.

The correlation functions between the several red components and the UV continuum are given in Table 14 and shown in Fig. 14. For all lines (except $\operatorname{MgII} \lambda 2798$ ) the $C C F$ peak indicate a mean delay of $-4 \pm 70$ days indicating no delay at the resolution available in the sampling and consistent with the absence of delay indicated by the $D C F$. Since for CIV, SiIV and NV the red component disappears completely when the continuum is weak, the center of $C C F$ is the only meaningful parameter for the delay determinations. At very low levels no line compo- nent flux can be determined and the lightcurves of the continuum and the line become very unequally sampled, completely distorting the extremes of the $C C F$. Therefore the centroid of the $C C F$, at $\approx-200$ days, becomes solely a representation of the incompleteness of the light curve sampling and has no physical meaning. On the other hand, this component is the only one for which a statistically significant TF could be determined. The TF shows an unresolved peak at zero days delay and no additional side lobes at larger delays as seen in the full line intensities (see also Sect. 4.2). The resulting Echo Map was fully consistent in the reproduction of the continuum lightcurve and was statistically quite stable. Although the errors are large, a weak correlation for the red component line ratios of $\operatorname{Ly} \alpha \lambda 1216 / \mathrm{CIV} \lambda 1549$ and $\operatorname{Ly} \alpha \lambda 1216 / \operatorname{MgII} \lambda 2798$ is clearly present at lower levels of the UV continuum brightness (Fig. 20), again appearing to flatten at levels above $>1510^{-14} \mathrm{erg} \mathrm{s}^{-1} \mathrm{~cm}^{-2} \AA^{-1}$. The mean values are $\mathrm{Ly} \alpha / \mathrm{CIV}=4 \pm 2, \mathrm{Ly} \alpha / \mathrm{NV}=4 \pm 1, \mathrm{Ly} \alpha / \mathrm{SiIV}=5 \pm 2$ and $\operatorname{Ly} \alpha / \mathrm{MgII}=10 \pm 6$.

\subsubsection{The blue component}

The blue component has a mean FWHM of $\approx 5900 \mathrm{~km} \mathrm{~s}^{-1}$ and a velocity with respect to the narrow line of $\approx-3600 \mathrm{~km} \mathrm{~s}^{-1}$. Its contribution to the total intensity is $19,23,17$ and $26 \%$ for $\operatorname{Ly} \alpha \lambda 1216, \operatorname{CIV} \lambda 1549$, $\operatorname{MgII} \lambda 2798$ and SiIV $\lambda 1397$, respectively, generally lower than the red component. The intensity correlation with the UV continuum (Fig. 15) is intermediate between the red and the central component for all lines but $\operatorname{MgII} \lambda 2798$ which is constant. It does not correlate as directly with the UV continuum as the red component but it does not show the strong loops shown by the central component. The amplitude of its variations (Table 7) does not vary significantly between Ly $\alpha \lambda 1216$, CIV $\lambda 1549$ and SiIV $\lambda 1397$.

The correlation functions for blue component with the UV continuum are shown in Fig. 16 and given in Table 14 . The correlation functions for the blue component are significantly different from those for both the red and central components and consistent between the $C C F$, for which the center measure gives a mean delay of $230 \pm 95$ days, and the $D C F$, which results in a delay of 200 days (Fig. 16).

For the blue component the line ratios (Fig. 19) show a strong correlation with the continuum for both Ly $\alpha / \mathrm{CIV}$ and $\operatorname{Ly} \alpha / \mathrm{MgII}$, while $\operatorname{Ly} \alpha / \mathrm{SiIV}$ keeps constant within the errors. The mean values are $2 \pm 1$ for $\operatorname{Ly} \alpha / \mathrm{CIV}, 5 \pm 2$ for Ly $\alpha / \mathrm{MgII}$ and $10 \pm 8$ for Ly $\alpha / \mathrm{SiIV}$. The H $\beta \lambda 4861$ decomposition by Wamsteker et al. (1985) required a very broad component (of $F W H M=9220 \mathrm{~km} \mathrm{~s}^{-1}$ and velocity respect to the narrow line of $-1710 \mathrm{~km} \mathrm{~s}^{-1}$ ). Depending on the line ratios of the red and blue component identified in the UV spectra with respect to $\mathrm{H} \beta \lambda 4861$ it is quite probable that this is essentially the optical counterpart of these two components in the UV lines. A similar 
result was also found for the line decomposition applied to the simultaneous UV and optical spectra of NGC 5548 (Wamsteker et al. 1990), where a very broad component clearly present in the UV was only marginally detectable in the $\mathrm{H} \beta \lambda 4861$ profile. Lub \& De Ruiter (1992) also obtain a very broad and variable component in $\mathrm{H} \alpha \lambda 6563$ and $\mathrm{H} \beta \lambda 4861$, in order to explain the different delays shown for these lines with respect to the optical variable continuum of $\approx 400$ days for $\mathrm{H} \alpha \lambda 6563$ and $270 \pm 150$ days for $\mathrm{H} \beta \lambda 4861$. For the blue component also no solution could be obtained for the transfer function at a reasonable level of significance, most likely also associated with the nonlinearity in the line response as was the case for the central component.

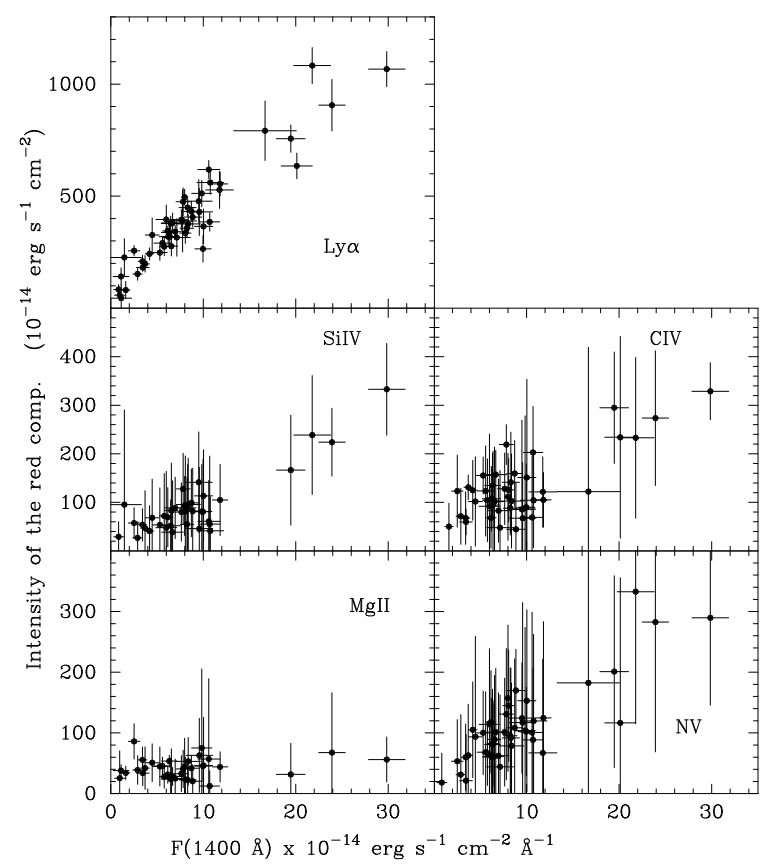

Fig. 13. Intensities of the red components (see Table 9) versus the UV continuum at $1400 \AA$

\section{Physical conditions of the gas: Photoionization models}

The strong correlations obtained between the intensity of the different broad components and the UV flux, confirms that the main excitation mechanism of the gas is photoionization by a central source. To obtain better insight in the physical conditions and the spatial relations of these parts of the BLR which appear to constitute separate regions, we have made separate photo-ionization calculations for each of the regions to compare with the results described in the previous section.

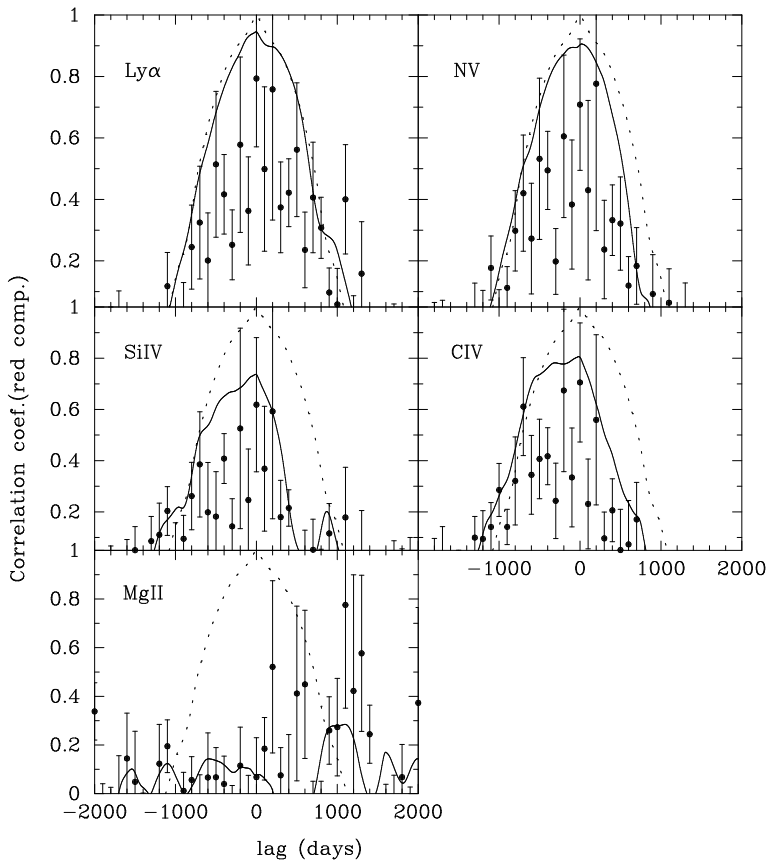

Fig. 14. Correlation Functions for the red component (see also Table 14). Solid line: Cross Correlation Function (CCF). Points and error bars: Discrete Correlation Function (DCF). Dotted line: Autocorrelation Function (ACF) for the UV continuum at $1400 \AA$

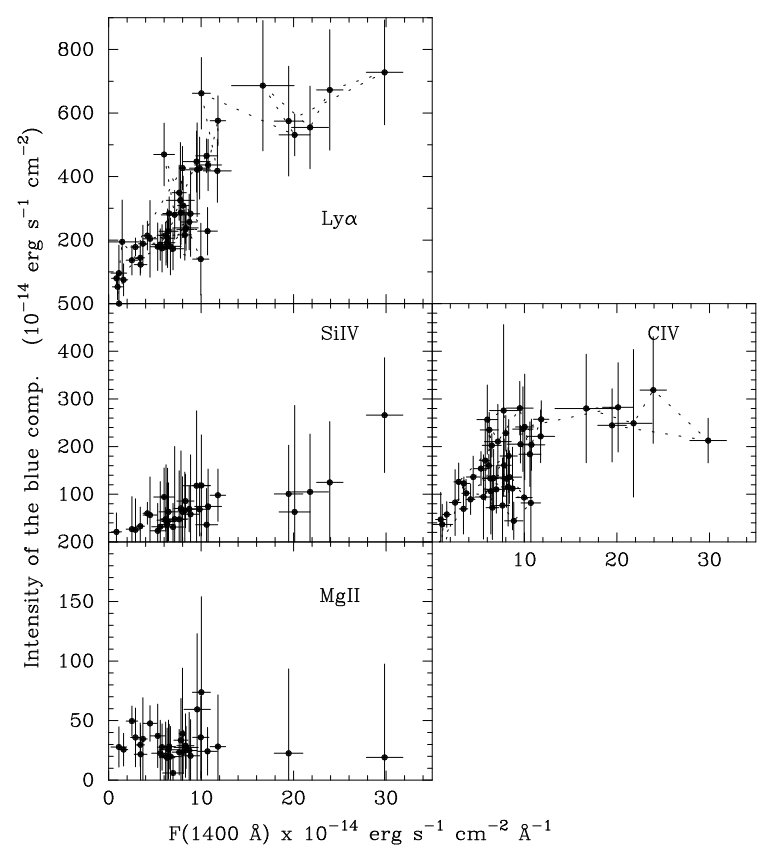

Fig. 15. Intensities of the blue components versus the UV continuum at $1400 \AA$ 


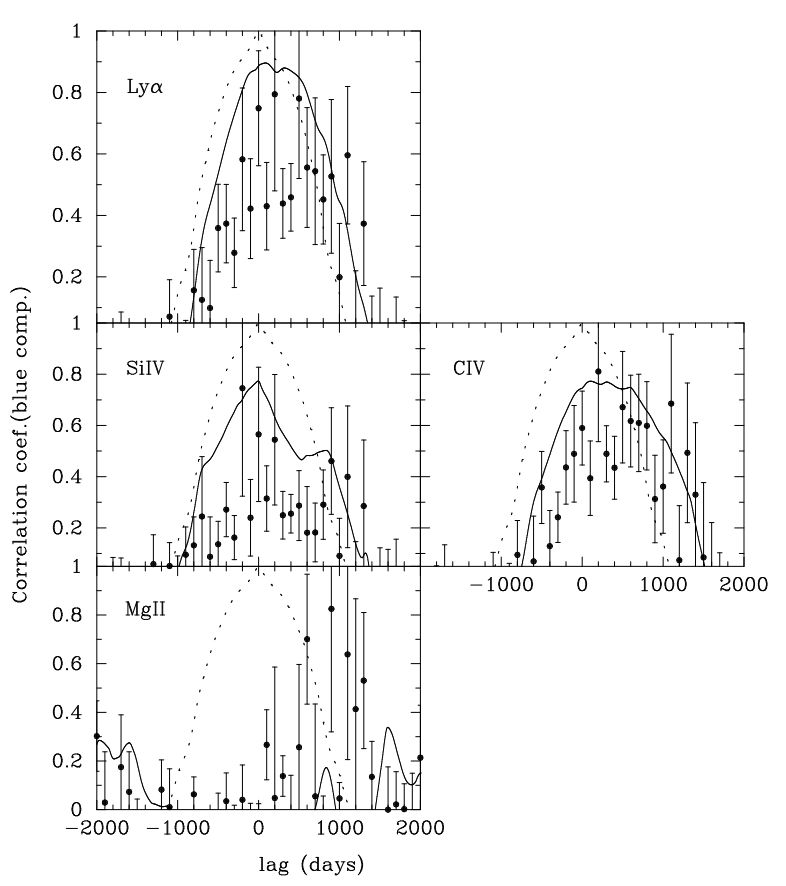

Fig. 16. Correlation functions for the blue components (see Table 14). Solid line: Cross Correlation Function (CCF). Points and error bars: Discrete Correlation Function (DCF). Dotted line: Autocorrelation Function (ACF) for the UV continuum at $1400 \AA$

We used the photo-ionization code CLOUDY (Ferland 1991: CLOUDY 80.06). The initial parameters needed by CLOUDY are: the shape and intensity of the incident continuum $(14<\log \nu<20)$, the chemical composition, the distribution of the gas (radial extension and the run of density with radius) and the covering factor. We have assumed solar composition, an open (small radiative covering factor) spherical geometry and constant density. For the ionizing continuum we have used the observed continuum derived in Sect. 3.3 at four levels of brightness (Fig. 5), corresponding to the $F(1400 \AA$ ) of 23.27 (high), 10.04 (high-intermediate), 3.78 (low-intermediate) and $1.3110^{-14} \mathrm{erg} \mathrm{s}^{-1} \mathrm{~cm}^{-2} \AA^{-1}$ (low), respectively. To connect the frequency gap between the Radio and the FIR range, linear interpolation has been used. To account for the big blue bump, the optical-UV continuum has been parametrized with a power law $F_{\nu}=F_{0} \times \nu^{-0.07} \times \mathrm{e}^{-\nu / \nu_{\text {cut }}}$, fitting $F_{0}$ to the observed $F(1400 \AA)$ for each level. The cut-off energy is a critical factor in the input spectrum. Clavel et al. (1990) and Binette et al. (1989) suggested 0.83 and 0.73 ryd, respectively, based on photoionization models for Fairall-9. The simultaneous observation with IUE, ROSAT and GINGA (Fig. 5c) shows however that such a cut-off frequency does not correspond with the observed instantaneous spectrum of Fairall-9. Although it might be possible that the spectrum incident on the BLR is different from that which we observe, at this stage we have no convincing arguments to apply a spectrum different from the observed one. Then, a considerably slower drop off is indicated and a cut-off at $3.5 \mathrm{ryd}$ as shown in Fig. 17 was applied. In the X-rays and higher frequencies we used a power law similar to the UV-optical one, with a spectral index $\alpha=-0.96$ and an arbitrary cut-off at $0.1 \mathrm{MeV}$ (consistent with the $\gamma$ ray observations of other Seyfert 1 nuclei with CGRO). The constant was defined by the observed integrated flux at $2-10 \mathrm{keV}$. To bring all in an absolute scale we used $H_{0}=100 \mathrm{~km} \mathrm{~s}^{-1} \mathrm{Mpc}^{-1}$ and all fluxes have been corrected for redshift with $z=0.0461$. We have calculated models with a range of total hydrogen density of the gas, $n$, between $10^{7}$ and $10^{12} \mathrm{~cm}^{-3}$ and for the total hydrogen column density, $N_{\text {col }}$, a range between $10^{21}-10^{26} \mathrm{~cm}^{-2}$. The inner radius of the gas cloud, $r$, was chosen over a range from 50 to 600 light-days, consistent with the delays obtained for the BLR line components with respect to the UV continuum derived in Sect. 4.3.

With these initial conditions many models have been calculated, obtaining from them the line luminosity for a geometric covering factor of $1(\Omega / 4 \pi=1)$. Comparing the results of the models for this value of $\Omega / 4 \pi$ with the observed luminosities, the real covering factor can be obtained. The ionization parameter, $U$, is determined from the incident continuum luminosity, the density and the distance assumed for each model. So, comparing the relative intensities of the lines obtained from the models with the observed ones, the best fitting model can be found.

\subsection{Comparison with the observations}

To compare the models and the observations we have used the observed line ratios for the components for $\operatorname{Ly} \alpha \lambda 1216 / \operatorname{CIV} \lambda 1549, \operatorname{Ly} \alpha \lambda 1216 / \operatorname{SiIV} \lambda 1397$ and $\operatorname{Ly} \alpha \lambda 1216 / \operatorname{MgII} \lambda 2798$, imposing that one single model fits all three line ratios, at all brightness levels of the continuum. Since the results for all models with $N_{\text {col }}=10^{25}$ and $10^{26} \mathrm{~cm}^{-2}$ are the same, we only consider the first. The conclusions for each broad component are:

\subsubsection{The central component}

The three line ratios are well described by models with the following characteristics distance (light-days); $N_{\text {col }}\left(\mathrm{cm}^{-2}\right) ; n\left(\mathrm{~cm}^{-3}\right)$ :

$50-10^{25}-10^{12} ; 100-10^{25}-10^{11} ; 150-10^{24-25}-10^{10}$; $200-10^{25}-10^{10} ; 250-10^{24-25}-10^{10}$.

The results for these best fitting models are shown in Fig. 18. Since $\operatorname{Ly} \alpha /$ CIV is nearly independent of the distance, also models with inner radius up to 600 light-days could be allowed too, but at these distances the other two ratios are too large at some continuum levels, restricting the distance range to $50-250$ light-days. We see that all models are consistent with the observed values of 


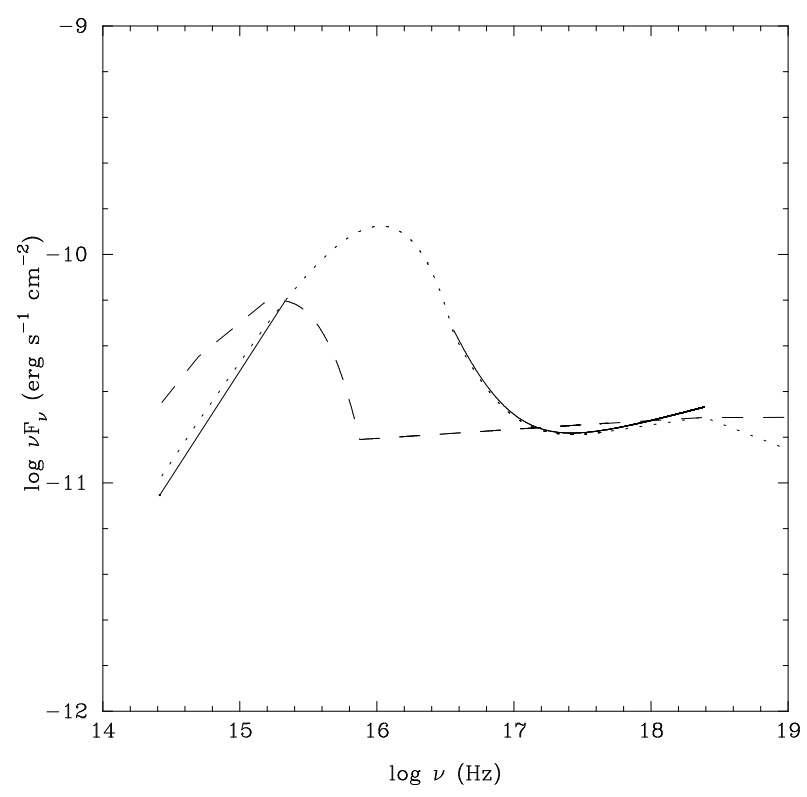

Fig. 17. Fit to the simultaneous observation IUE-ROSAT-GINGA. Solid line: Observation (between the UV continuum and the $J$ band $\alpha=-0.07$ has been used). Dotted line: Fit with a cut-off energy of 3.5 rydberg used as input for the photoionization models. Scratched line: SED with a 0.63 rydberg cut-off energy. It is clear from these simultaneous observations that the $0.73-0.83$ rydberg cut-off energy proposed by Binette et al. (1989) and Clavel \& Santos-Lleó (1990) is incompatible with the data

$\operatorname{Ly} \alpha / \operatorname{SiIV}$ and Ly $\alpha / \mathrm{MgII}$, but underestimate $\operatorname{Ly} \alpha / \mathrm{CIV}$, at bright continuum levels, since they converge to 1 . The mean ratio of $\operatorname{Ly} \alpha / \mathrm{H} \beta$, which increases with distance, for distances of 50, 100, 150, 200 and 250 light-days is, respectively, 12.6, 17.0, 21.7, 26.1 and 36.1. The ratio $\mathrm{Ly} \alpha / \mathrm{H} \beta$ for the central component estimated from the data of Wamsteker et al. (1985) is $\approx 13$ (Sect. 4.3.3), corresponding to models with radius of 50 and 100 light-days.

We show in Fig. 21 a comparison between the spectrum derived from the models and the individual line components with the observed mean spectrum. The mean emission lines only- spectrum shown in Fig. 21 has been corrected by $z=0.0461$ and corresponds to an observed $F(1400 \AA)$ of $\approx 8.510^{-14} \mathrm{erg} \mathrm{s}^{-1} \mathrm{~cm}^{-2} \AA^{-1}$ and to a Ly $\alpha$ central component luminosity of $\log L\left(\mathrm{erg} \mathrm{s}^{-1}\right)=$ 43.030. The comparison in Fig. 21 is thus for an highintermediate level of the continuum, with an observed value $F(1400 \AA)=10.0410^{-14} \mathrm{erg} \mathrm{s}^{-1} \mathrm{~cm}^{-2} \AA^{-1}$. For this level the models give $U=0.003,0.009,0.039,0.022$ and 0.014 and corresponding covering factors of $12 \%, 5 \%$, $4 \%, 4 \%$ and $3 \%$ for distances of 50,100, 150, 200 and 250 light-days, respectively. These model spectra are similar, except perhaps the 50 light-days model, which produces a weak CII $\lambda 1335$, which is not observed. The SiIV $\lambda 1397$

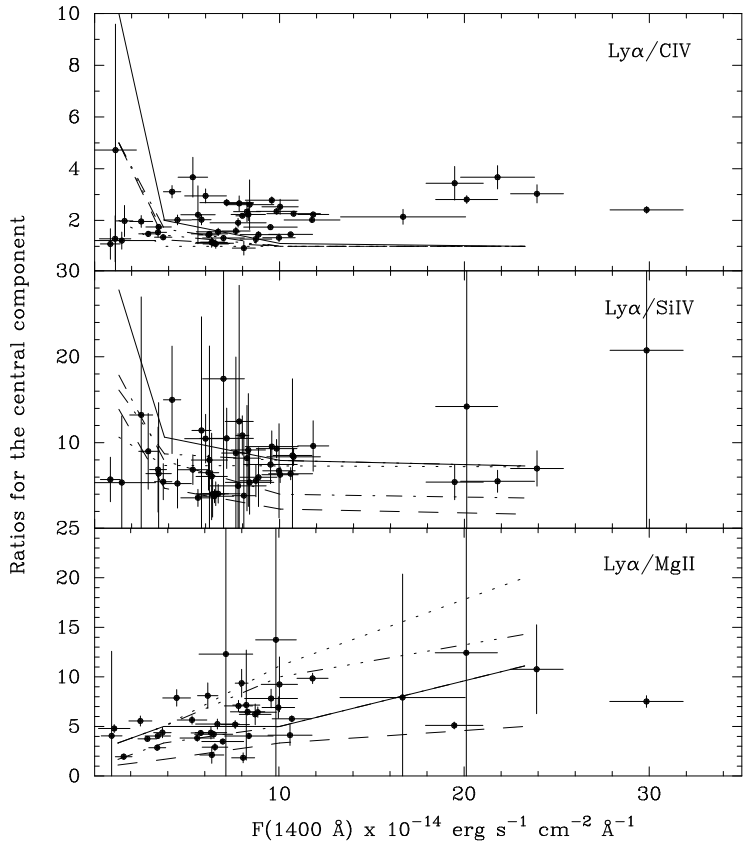

Fig. 18. Line ratios for the central component and the corresponding photoionization models. The different models are drawn with several types of lines over the observed ratios: distance (light-days) $-N_{\mathrm{col}}\left(\mathrm{cm}^{-2}\right)-n\left(\mathrm{~cm}^{-3}\right)=50-10^{25}-10^{12}$ $(---), 100-10^{25}-10^{11}(\cdot-\cdot-), 150-10^{24-25}-10^{10}$ $(\cdots), 200-10^{25}-10^{10}(\cdots-\cdots)$ and $250-10^{24-25}-10^{10}$ $(-)$

and HeII $\lambda 1640$ intensities are somewhat higher than observed. In Fig. 21a we show the mean spectrum together with the model of the central component for 100 lightdays, $N_{\text {col }}=10^{25} \mathrm{~cm}^{-2}$ and $n=10^{11} \mathrm{~cm}^{-3}$ model. The photoionization models with CLOUDY confine the region where the central component is produced to a distance from the ionizing source of $50-250$ light-days, with a column density of $N_{\text {col }}=10^{24-26} \mathrm{~cm}^{-2}$, an hydrogen density of $10^{12-10} \mathrm{~cm}^{-3}$ and a covering factor of $12-3 \%$, with $U$ between 0.003 and 0.039 (Table 14). The distance derived from the models is thus consistent with the lower end of the range derived from the delay of this component with respect to the UV continuum, $400 \pm 100$ light-days.

\subsubsection{The blue component}

The acceptable models for the observed ratios of this component are similar in their parameters range to the central component ones. They are characterized by (as above in Sect. 5.1.1):

$50-10^{23-24-25}-10^{11} ; 100-10^{23-24-25}-10^{10} ; 150-$ $10^{23-24-25}-10^{10} ; 200-10^{23-24-25}-10^{10}$ and $250-$ $10^{23-24-25}-10^{10}$.

Larger distances are excluded because they result in a too high $\operatorname{Ly} \alpha /$ SiIV ratio. The results for these models 
Table 14. Resulting parameters for the broad components

A) CENTRAL COMPONENT:

1. Correlation functions: Delays with respect to $F(1400 \AA)$ :

\begin{tabular}{|c|c|c|c|}
\hline \hline & \multicolumn{2}{|c|}{ CCF } & DCF \\
LINES & Peak & Center & Peak (interval =100 days) \\
\hline Ly $\alpha \lambda 1216$ & $212 \pm 90$ & $368 \pm 90$ & 500 \\
\hline CIV $\lambda 1549$ & $552 \pm 90$ & $500 \pm 90$ & 500 \\
\hline SiIV $\lambda 1397$ & $196 \pm 75$ & $236 \pm 75$ & 200 \\
\hline MgII $\lambda 2798$ & $-28 \pm 150$ & $482 \pm 150$ & 600 \\
\hline \hline
\end{tabular}

2. Photoionization models: Distance $=50-250$ light-days.

$N_{\text {col }}=10^{24-26} \mathrm{~cm}^{-2} \cdot n=10^{12-10} \mathrm{~cm}^{-3}$

Covering fac. $=12-3 \% . U=0.003-0.039$.

3. Reverberation analysis: Non-linearity in the response suggested.

B) RED COMPONENT:

1. Correlation functions: Delays with respect to $F(1400 \AA)$ :

\begin{tabular}{|c|c|c|c|}
\hline \hline \multirow{2}{*}{ LINES } & \multicolumn{2}{|c|}{ CCF } & DCF \\
\hline Ly $\alpha \lambda 1216$ & $-4 \pm 70$ & $-20 \pm 70$ & 0 \\
\hline CIV $\lambda 1549$ & $-16 \pm 70$ & $-208 \pm 70^{*}$ & 0 \\
\hline SiIV $\lambda 1397$ & $-12 \pm 70$ & $-222 \pm 70^{*}$ & 0 \\
\hline MgII $\lambda 2798$ & \multicolumn{2}{|c|}{ Not significant } & Not significant \\
\hline NV $\lambda 1240$ & $16 \pm 65$ & $-192 \pm 65^{*}$ & $200,0,-200$ \\
\hline \hline
\end{tabular}

2. Photoionization models: Distance $=50-250$ light-days.

$N_{\text {col }}=10^{24-26} \mathrm{~cm}^{-2} \cdot n=10^{11-10} \mathrm{~cm}^{-3}$.

Covering fac. $=6-3 \% . U=0.009-0.039$.

3. Reverberation analysis:Linear response suggested; Echo Map strongly peaked at zero delay without secondary peaks.

C) BLUE COMPONENT:

1. Correlation functions: Delays with respect to $F(1400 \AA)$ :

\begin{tabular}{|c|c|c|c|}
\hline \hline \multirow{2}{*}{ LINES } & \multicolumn{2}{|c|}{ CCF } & DCF \\
\hline Ly $\alpha \lambda 1216$ & $84 \pm 80$ & $204 \pm 80$ & Peak (interval $=100$ days) \\
\hline CIV $\lambda 1549$ & $104 \pm 100$ & $370 \pm 100$ & 200 \\
\hline SiIV $\lambda 1397$ & $-8 \pm 100$ & $120 \pm 100$ & $-200,0,200$ \\
\hline MgII $\lambda 2798$ & \multicolumn{2}{|c|}{ Not significant } & Not significant \\
\hline \hline
\end{tabular}

2. Photoionization models: Distance $=50-250$ light-days

$N_{\text {col }}=10^{23-26} \mathrm{~cm}^{-2} \cdot n=10^{11-10} \mathrm{~cm}^{-3}$.

Covering fac. $=5-2 \% . U=0.089-0.014$.

3. Reverberation analysis: Non-linearity in the response suggested.

* = These negative delays are due to the fact that the red component of the lines become too weak to measure when the UV continuum is very weak. With the uneven brightness distribution in the data set used here (Fig. 1), this maintains the peak value of the $C C F$, but introduces a strong asymmetry in the $C C F$ shape (see Fig. 14).

are shown with the observed ratios in Fig. 19. Similarly to the central component, all models reproduce the observed $\operatorname{Ly} \alpha \lambda 1216 / \operatorname{SiIV} \lambda 1397$ and $\operatorname{Ly} \alpha \lambda 1216 / \operatorname{MgII} \lambda 2798$ ratios very well, but underestimate Ly $\alpha \lambda 1216 / \operatorname{CIV} \lambda 1549$. The mean spectrum has in the blue component a Ly $\alpha$ luminosity of $\log L\left(\mathrm{erg} \mathrm{s}^{-1}\right)=42.833$. For the comparable high-intermediate level of the continuum, the models have $U=0.035,0.089,0.039,0.022$ and 0.014 and predict a covering factor of $5 \%, 2.9 \%, 2.5 \%, 2.3 \%$ and $2.1 \%$ for 50, 100, 150, 200 and 250 light-days, respectively. Compared with the mean spectrum, the spectra of the five models are very similar, and we show the 100 lightdays, $N_{\text {col }}=10^{23-24-25} \mathrm{~cm}^{-2}$ and $n=10^{10} \mathrm{~cm}^{-3}$ model for the blue component with the observed mean spectrum (Fig. 21b).

In summary, the models indicate that the gas producing the blue component of Ly $\alpha$, CIV, SiIV and MgII is situated at a distance of the ionizing source of $50-$ 250 light-days, with a column density of $10^{23-26} \mathrm{~cm}^{-2}$, an hydrogen density of $10^{11-10} \mathrm{~cm}^{-3}$ and a covering factor of $5-2 \%$, where $U$ is confined between 0.089 and 0.014 . This range of parameters is similar to the central component one (Table 14). The distance derived from the photoionization model for the blue component is consistent with the mean delay of this component respect to the UV continuum ( $230 \pm 95$ days).

\subsubsection{The red component}

The models, which fit best to the observed ratios for this component are characterized by (as above in Sect. 5.1.1): $50-10^{25}-10^{11} ; 100-10^{25}-10^{11} ; 150-10^{24-25}-10^{10}$; $200-10^{25}-10^{10}$ and $250-10^{24-25}-10^{10}$. Also here larger distances are excluded because they result in an 


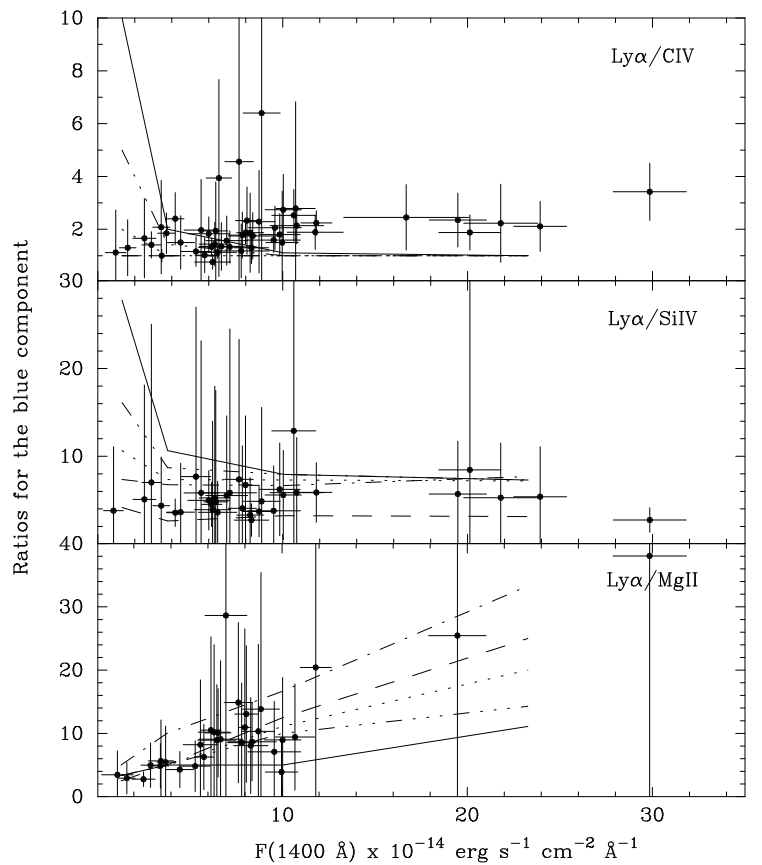

Fig. 19. Line ratios for the blue component and the corresponding photoionization models. The different models are drawn with several types of lines over the observed ratios: distance (light-days) $-N_{\text {col }}\left(\mathrm{cm}^{-2}\right)-n$ $\left(\mathrm{cm}^{-3}\right)=50-10^{23-24-25}-10^{11}(---), 100-10^{23-24-25}-10^{10}$ $(\cdot-\cdot-), 150-10^{23-24-25}-10^{10}(\ldots), 200-10^{23-24-25}-10^{10}$ $(\cdots-\cdots)$ and $250-10^{23-24-25}-10^{10}(-)$

overestimate the $\operatorname{Ly} \alpha /$ SiIV ratio. These models are shown in Fig. 20 with the observed ratios. All models fit perfectly to $\operatorname{Ly} \alpha \lambda 1216 / \operatorname{SiIV}$ and $\operatorname{Ly} \alpha \lambda 1216 / \operatorname{MgII} \lambda 2798$, but Ly $\alpha \lambda 1216 / \mathrm{CIV} \lambda 1549$ is always underestimated.

For this component we have been able to isolate also the $\operatorname{Ly} \alpha \lambda 1216 / \mathrm{NV} \lambda 1240$ ratio. Its observed value is low (between 2 and 8) which can only be explained with models that, at similar distances, have an order of magnitude lower hydrogen density and a very small column density $\left(10^{21} \mathrm{~cm}^{-2}\right)$. This might indicate that the BLR is composed of optically thin clouds besides of the optically thick ones considered above, which might also explain better the $\operatorname{Ly} \alpha /$ CIV ratio, as have been recently suggested by Shields et al. (1995). However, even though the previous models (optically thick) seem to underestimate the NV $\lambda 1240$ line strength, we will use them since at the required low column density no consistent solution was feasible for all other line ratios. The mean spectrum has a red component Ly $\alpha$ luminosity of $\log L\left(\mathrm{erg} \mathrm{s}^{-1}\right)=42.954$. For the high-intermediate level of the continuum, the models have $U=0.035,0.0089,0.039,0.022$ and 0.014 and a covering factor of $6 \%, 4 \%, 3.3 \%, 3.0 \%$ and $2.9 \%$ for 50 , 100, 150, 200 and 250 light-days, respectively. Comparison with the mean spectrum shows that the model spectra

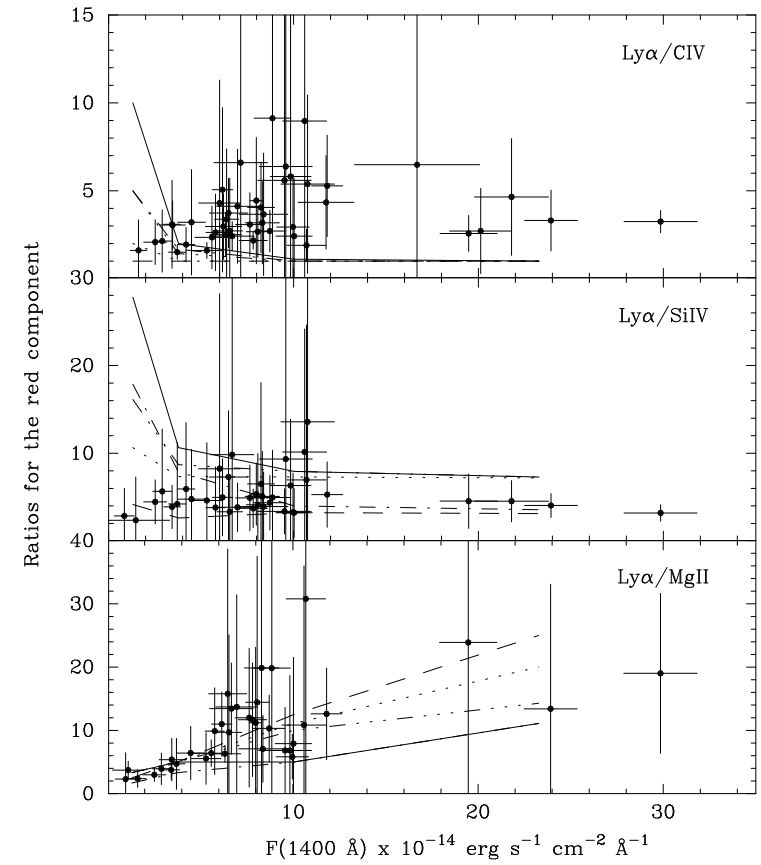

Fig. 20. Lines ratio for the red component and the corresponding photoionization models. The different models are drawn with several types of lines over the observed ratios: distance (light-days) $-N_{\mathrm{col}}\left(\mathrm{cm}^{-2}\right)-n\left(\mathrm{~cm}^{-3}\right)=50-10^{25}-10^{11}$ $(---), 100-10^{25}-10^{11}(\cdot-\cdot-), 150-10^{24-25}-10^{10}$ $(\cdots), 200-10^{25}-10^{10}(\cdots-\cdots)$ and $250-10^{24-25}-10^{10}$ (-)

are very similar, and in Fig. 21c the model shown is for 100 light-days, $N_{\text {col }}=10^{25} \mathrm{~cm}^{-2}$ and $n=10^{11} \mathrm{~cm}^{-3}$. In summary, the photoionization model calculations indicate that the gas producing the red component of Ly $\alpha \lambda 1216$, $\operatorname{CIV} \lambda 1549$, SiIV $\lambda 1397$ and $\operatorname{MgII} \lambda 2798$ is situated at a distance of the ionizing source of $50-250$ light-days, with $N_{\text {col }}=10^{24-26} \mathrm{~cm}^{-2}, n=10^{11-10} \mathrm{~cm}^{-3}$ and a covering factor of $6-3 \%$ with $U$ between 0.009 and 0.039 (Table 14).

The models alone do not allow to distinguish the gas producing the red component from that producing the blue component, because the same parameter range is applicable for both (and very similar to those for the central component as well). On the other hand the delay of the red component of $<50$ light-days with respect to the continuum falls outside the range suggested by the models, in contrast to the results found for the central and blue component. One possible explanation for this is that the gas emitting the red component is situated along the line of sight to the observer or that the regions producing both the red and the blue component are at the same distance falling in toward the central source (see Sect. 7 for a more detailed explanation). 
The difficulty associated with the $\operatorname{Ly} \alpha / \mathrm{CIV}$ ratio for all models can be explained if: (a) The carbon abundance is less than the solar one (see the next section); or (b) The continuum shape in $\log \left(\nu F_{\nu}\right)$ between 13.6 and $48 \mathrm{eV}$ $(\log \nu=15.5$ and 16 , respectively) is not as steep as the exponential cut-off used for the input continuum spectrum (Fig. 17), so that the fraction of ionizing photon density to which CIV responds, decreases with respect to the photon density which Ly $\alpha$ responds, keeping in mind that the cut-off frequencies suggested by Clavel \& Santos-Lleó (1990) and Binette et al. (1989) are inconsistent with the observed soft X-rays excess (Fig. 17); or (c) There is not a smooth connection between the UV and the soft X-rays, with an absorption in the continuum between $48-150 \mathrm{eV}$, resulting in an increase in the Ly $\alpha /$ CIV ratio. Such absorption can be present if optically thin material, transparent at $\leq 50 \mathrm{eV}$ and absorbing at $50-500 \mathrm{eV}$, is present between the ionizing source and the BLR (Ferland et al. 1990); or (d) It is necessary to introduce in the models two populations of clouds: optically thin and optically thick ones, as it has been shown by Shields et al. (1995) to be possible for the specific case of F-9.

\section{Relation gas-dust in the BLR}

The delay obtained for the central component with respect to the UV continuum of $400 \pm 100$ days is similar to that obtained for the $K$ and $L$ bands, where the emission is caused by heated dust (Clavel et al. 1989; Sect. 4.3.3). Therefore the dust, and the gas producing the central component, seem to be coexisting in the same region. Even, if the central gas is situated at $\approx 200$ light-days (more compatible with the photoionization models) and the dust at $400 \pm 100$ light-days, it would appear that the outer part of the gas would be (within the errors) associated with the inner part of the dust zone. An upper limit to the dust mass at 400 light-days has been estimated by Clavel et al. in $0.02 M_{\odot}$ and we will estimate the mass-to-dust ratio from the maximum luminosity of both CIV $\lambda 1549$ and $\mathrm{H} \beta \lambda 4861$. Assuming a typical $N_{\mathrm{e}}=10^{9} \mathrm{~cm}^{-3}$ and that at the CIV emission maximum all carbon is $\mathrm{C}^{+3}$ and all ions are in the lower level, $N_{1}=N(\mathrm{C})$, with a maximum of $L_{21}($ central $)=1.03410^{43} \mathrm{erg} \mathrm{s}^{-1}, N(\mathrm{C})=1.2510^{54}$. For a solar carbon abundance of $\mathrm{C} / \mathrm{H}=4.710^{-4}$ this gives $N(\mathrm{H})=2.6610^{57}$ and $M=2.24 M_{\odot}$ (Aller 1984). Then, the ratio mass (central gas) $/ \operatorname{mass}$ (dust) $=100$, similar to the galactic value of 200 . Using the $\mathrm{H} \beta \lambda 4861$ luminosity, $L=N\left(\mathrm{H}^{+}\right) N_{\mathrm{e}} \alpha h \nu$ (Aller 1984), for the Case B of recombination $\left(\alpha \times h \nu=0.86310^{-25} \mathrm{erg} \mathrm{cm}^{3} \mathrm{~s}^{-1}\right)$, with $N_{\mathrm{e}}=10^{9} \mathrm{~cm}^{-3}$ and the maximum $\mathrm{H} \beta \lambda 4861$ luminosity of $L($ central $)=1.5210^{42} \mathrm{erg} \mathrm{s}^{-1}$ (Wamsteker et al. 1985), we find $N\left(\mathrm{H}^{+}\right)=1.7710^{58}$. Then $M=15 M_{\odot}$ and the ratio mass (central gas) $/$ mass (dust) $=750$, four times larger than the galactic value.

Note that the mass of the central gas is in the range of $2-15 M_{\odot}$ from the different lines and the subsequent

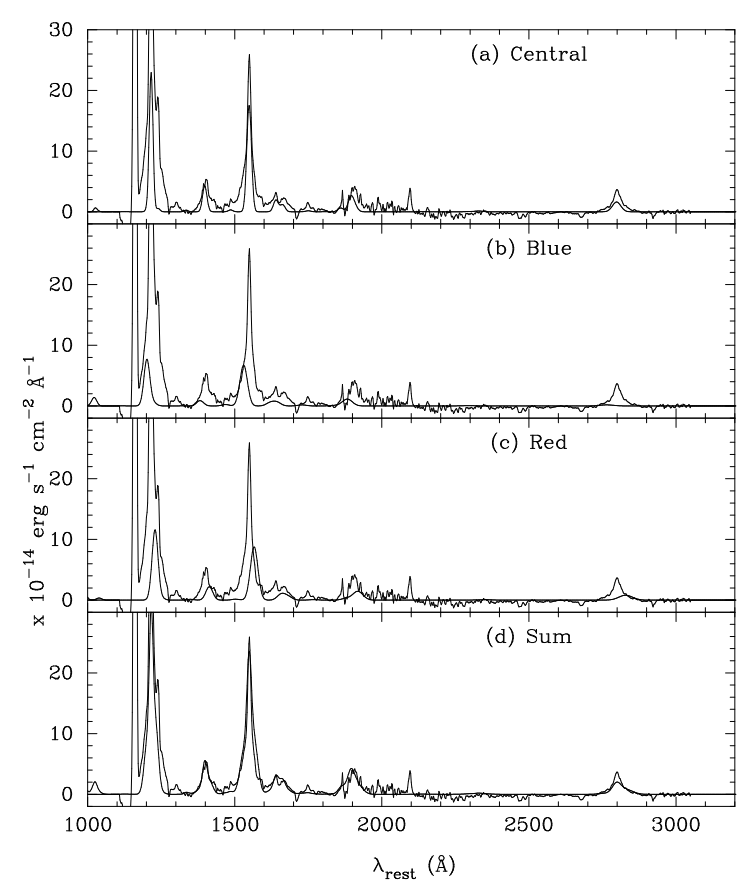

Fig. 21. Observed mean spectrum and the obtained spectra from the photoionization models for each component. a) The observed mean spectrum and the model with 100 light-days, $N_{\text {col }}=10^{25} \mathrm{~cm}^{-2}$ and $n=10^{11} \mathrm{~cm}^{-3}$ for the central component. b) The observed mean spectrum and the model with 100 light-days, $N_{\text {col }}=10^{23-24-25} \mathrm{~cm}^{-2}$ and $n=10^{10} \mathrm{~cm}^{-3}$ for the blue component. c) Equal to the central component for the red one. d) The sum of each model for the different components over the observed mean spectrum. Take into account that the narrow component has not been fitted

mass(central gas)/mass(dust) ratio extends from 100 to 750 could indicate that the abundance $\mathrm{C} / \mathrm{H}$ is less than the solar one. If we take $N(\mathrm{C})=1.2510^{54}$ from the first calculus and $N\left(\mathrm{H}^{+}\right)=1.7710^{58}$ from $\mathrm{H} \beta \lambda 4861$, an abundance $\mathrm{C} / \mathrm{H}=710^{-5}$ is obtained, an order of magnitude lower than the solar abundance assumed previously. Another explanation to the difference in the results from both lines might be that also the mixture of gas-to-dust is stratified, as had been previously found for the gas only. This is a consequence of the significantly different derived gas mass depending on the ionization state of the ion used for the calculation, while the dust can not survive in the inner regions. This would be consistent with the general concept from previous results suggesting stratification in the BLR (e.g. Clavel et al. 1991). However at the same time the coexistence of the gas and dust, and the difference in line delays with respect to the continuum, seem to indicate that we are most likely dealing with a situation where the mixture with dust and the ionization stratification are all taking place in a region at 
approximately $300-400$ light-days from the central source in an anisotropic radiation field.

\section{Structure and dynamic of the BLR}

With the results obtained above from the behavior of the broad-line components we will try to derive the structure of the BLR in some detail, under the assumption that the gas producing these components is gravitationally bound. Then the mass of the central object (the black hole in the standard theory) can be calculated, as usual, from $M=v^{2} r / G$, where $v$ is the gas velocity and $r$ its distance to the object. We find the distance can be estimated from the delays obtained with the correlation functions in combination with the distances deduced from the central photoionization models. To determine the velocity we assume that the main mechanism of line-broadening is macroscopic turbulent movement, implying that the mean velocity is $v_{\text {tur }}=0.6 \times F W H M$. For the central component this gives $v_{\text {tur }} \approx 2160 \mathrm{~km} \mathrm{~s}^{-1}$. Its distance to the central object is more difficult to establish, due to the fact that the one deduced from the $C C F, r \approx 400$ light-days, is twice as large as that from the photoionization models of $r \approx 200$ light-days. This could indicate some special geometry with respect to the observer, which will be discussed below. For the current discussion we will use a distance of $r \approx 200$ light-days, obtaining $M \approx 210^{8} M_{\odot}$, equal to the estimated one by Clavel et al. (1989). The similarity in these results is not too surprising since we find a smaller distance but the turbulent velocity of the individual component is compensating for this.

If we now use this value of $M$ and the $F W H M$ for the blue and the red components, distances of 75 and 77 light-days, respectively, are obtained. This $\approx 80$ light-days distance, equal for both components, is significantly different from the delay calculated from the correlation functions, $-4 \pm 70$ and $230 \pm 95$ days, respectively. This can be explained if infalling motion of the gas at a distance of $\approx 80$ light-days is considered, because then the red flux does not lag behind the continuum variations and the blue flux will be delayed by twice the light travel time (Koratkar \& Gaskell 1991). Koratkar \& Gaskell, applying cross correlations to the wings of the Fairall-9 lines, obtain for CIV $0 \pm 43$ days and $200 \pm 66$ days for the red and the blue flux, respectively, concluding that the gas producing these fluxes is at a distance of $100 \pm 44$ light-days and has an infall movement, compatible with our result. However, they obtain for MgII $\lambda 2798$ that both wings are delayed with respect to the continuum $200 \pm 50$ days, suggesting that the movement is chaotic or circular. We have not been able to analyze the wings of $\operatorname{MgII} \lambda 2798$ because the correlation functions are not significant due to the small variability amplitude of the $\operatorname{MgII} \lambda 2798$ flux with the continuum.

Supposing that the red and the blue components of Ly $\alpha$, CIV and SiIV are produced in the same zone of gas at $\approx 80$ light-days and is infalling, there is a problem if this region is spherically symmetric. In that case we should obtain central gas at the same distance, while we find that the central component originates further away. The absence of this central gas indicates a non-spherically symmetric or anisotropic continuum emission, so that the majority of the gas responsible for the red and the blue flux is along the line of sight of the observer (the angular extend of course limited by the time resolution of our data). Besides, the central component is produced at a larger distance, $r \approx 200$ light-days, explaining the derived twice larger delay if this region of gas is behind the source, suggesting that either the symmetry is non-spherical or the continuum emission is anisotropic, or both. The absence of central gas between the source and the observer could, in this context, be explained as due to the presence of dust in the non-illuminated or neutral part of the clouds, which would absorb its emission. Then the dust emitting in the near IR at $400 \pm 100$ light-days (Clavel et al. 1989) should extend between $\approx 200$ light-days and larger distances.

\section{Conclusions}

The analysis of the ultraviolet variability of the AGN in the Seyfert I galaxy Fairall-9 (continuum and emission lines) has been made using IUE spectra of this AGN between 1978 and 1991 (4903 days) with a mean sampling interval of 96 days. These data have been combined with those available at other wavelength domains to derive the SED of F-9. This instantaneous SED has been used as input for the ionizing continuum in photoionization models. The line profile variability has allowed the decomposition of the strongest lines in several components. The comparison between the line ratios in these components for Ly $\alpha \lambda 1216$, CIV $\lambda 1549$, SiIV $\lambda 1397$ and MgII $\lambda 2798$ combined with the photoionization model calculations and the results of reverberation analysis has allowed to derive a consistent model for the structure and dynamics of the BLR.

1. The UV continuum shows a dominant variation with $R_{\max }=33 \pm 4$ and a characteristic time of 182 days. Also faster variability on time scales of the order of 56 days appears present, but this has not been adequately sampled in the available data. The intrinsic spectral index UV-optical is $\alpha=-0.07 \pm 0.02$ and the optical variations don't lag behind the UV ones. In the NIR, The $J$ band flux shows as an extension of the UVoptical continuum with $\alpha_{J-\mathrm{UV}}=-0.07 \pm 0.05$. On the contrary, the $H, K$, and $L$ bands variations lag behind the UV ones (Clavel et al. 1989). The $2-10 \mathrm{keV}$ flux is proportional to the UV continuum at low flux levels, while at higher UV flux levels this proportionality breaks down. 
2. The SED of the F-9 nucleus shows two important flux excesses, extending over the FIR-NIR range and from the $J$-band to the X-rays. The FIR-NIR excess, together with the delays of the $H, K$ and $L$ bands fluxes with respect to the UV continuum, is consistent with reradiation from dust heated directly by the ionizing continuum, as already suggested by Clavel et al. (1989) and Barvainis (1992). The deduced hot dust mass is $0.02 M_{\odot}$ at a distance of $400 \pm 100$ light-days of the UV ionizing source (Clavel et al. 1989).

The problems for a simple disk model to explain the big blue bump ( $J$-soft X-rays) of F-9 (Clavel et al. 1989 and Courvoisier \& Clavel 1991) and the strong correlation observed in the X-rays-UV variations at low UV flux levels for Fairall-9, are similar to those reported for NGC 5548 (Clavel et al. 1992) and NGC 4151 (Perola et al. 1986). In these AGN the partial solution includes reprocessing on an accretion disk of the hard X-rays emitted from a region above the disk. The presence of strong $\mathrm{Fe} \mathrm{K} \alpha$ line centered at $6.63-6.64 \mathrm{keV}$ in the Ginga spectra of F-9 supports this model. However, the equivalent width of this line, $230-360 \mathrm{eV}$, could indicate that the $\mathrm{Fe}$ abundance is $\approx 3$ larger than the cosmic one (Zdziarski 1990; Bai 1979). Wills et al. (1985) similarly obtained this Fe overabundance in their Seyfert 1 galaxies sample from the high ratio FeII $($ optical $+\mathrm{UV}) / \operatorname{MgII} \lambda 2798 \approx 8$. This is consistent with the ratio obtained by us (see Table 8 ).

3. The line profile variability for $\operatorname{Ly} \alpha \lambda 1216, \operatorname{CIV} \lambda 1549$, SiIV $\lambda 1397$ and $\operatorname{MgII} \lambda 2798$ has allowed us to isolate the same four components (represented by gaussians) in all lines and at all levels of brightness: one narrow (i.e. unresolved at the IUE resolution), and three broad components: a central $\left(F W H M=3600 \mathrm{~km} \mathrm{~s}^{-1}\right.$, velocity same as the narrow line), a red shifted $\left(F W H M=5800 \mathrm{~km} \mathrm{~s}^{-1}, v=+3300 \mathrm{~km} \mathrm{~s}^{-1}\right)$ and a blue shifted $\left(F W H M=5900 \mathrm{~km} \mathrm{~s}^{-1}\right.$, $v=-3600 \mathrm{~km} \mathrm{~s}^{-1}$ ) one. The strong correlations obtained between the three broad components and the UV continuum indicates that photoionization is the dominant mechanism in the BLR. The delays between these components and the UV continuum are $400 \pm 100,-4 \pm 70$ and $230 \pm 95$ days for the central, red and blue one, respectively.

4. Photoionization models (CLOUDY) with a UV-optical cut-off of 3.5 ryd, derived from the observed instantaneous SED, have been applied to the observed lines ratios of the broad components, and indicate that the BLR is situated at 50-250 light-days from the ionizing source, with an hydrogen density of $10^{12-10} \mathrm{~cm}^{-3}$, a column density of $10^{23-26} \mathrm{~cm}^{-2}$ and a covering factor of $12-2 \%$, with an ionization parameter between 0.003 and 0.089. However, all these optically thick models underestimate the $\mathrm{Ly} \alpha / \mathrm{CIV}$ ratio and overestimate the Ly $\alpha / \mathrm{NV}$ one. The solution to this discrepancy could be associated with the co-existence of optically thick and optically thin material in the BLR, as suggested by Shield (1995).

5. From all the results we have propose a model for the structure and dynamics of the BLR: the mass of the central compact object is $M \approx 210^{8} M_{\odot}$. Around this exist two distinct gas zones within the BLR: the gas producing the central component at $\approx 200$ light-days and the one emitting the red and the blue fluxes at $\approx 80$ light-days moving inward to a central source. These results require that both gas zones be localized along the line-of-sight or, alternatively, that the continuum emission must be strongly anisotropic. Besides, the gas emitting the central component is most likely mixed with the dust and the central gas to dust mass ratio is Mass (central gas) $/ \operatorname{mass}($ dust $)=100-750$.

Acknowledgements. Part of this work was supported by a Comunidad de Madrid FPI grant between 1990-1994. M.C. Recondo-González thanks all the VILSPA personal for their kindness and Antonio De la Fuente for his help.

\section{References}

Aller L.H., 1984, Physics of Thermal Gaseous Nebulae, Astrophysics and Space Science Library

Bai T., 1979, Solar Phys. 62, 113

Barvainis R., 1992, ApJ 400, 502

Binette L., Prieto A., Szuszkiewicz E., Zheng W., 1989, ApJ 343, 135

Boggess A., et al., 1978a, Nat 275, 372

Boggess A., et al., 1978b, Nat 275, 377

Boggess A., Wilson R., 1989, in "Exploring the Universe with the IUE satellite", Kondo Y. (ed.) Astrophys. Space Sci. Library, p. 3

Bohlin R.C., Grillmair C.J., 1988, ApJSS 66, 209

Busko I.C., Steiner J.E., 1988, MNRAS 232, 525

Busko I.C., Steiner J.E., 1989, MNRAS 238, 1479

Carleton N.P., Elvis M., Fabbiano G., Willner S.P., 1987, ApJ 318,595

Carleton N.P., Elvis M., Fabbiano G., Willner S.P., Lawrence A., Ward M., 1987, ApJ 18, 595

Chapman G.N.F., Geller M.J., Huchra J.P., 1985, ApJ 297, 151

Clavel J., 1987, "International Ultraviolet Explorer Image Processing Information Manual", version 2.0 (new software) European Edition

Clavel J., Gilmozzi R., Prieto A., 1988, A\&A 191, 392

Clavel J., Wamsteker W., Glass I.S., 1989, ApJ 337, 236

Clavel J., Santos-Lleó M., 1990, A\&A 230, 3

Clavel J., Nandra K., Makino F., Pounds K., Reichert G.A., Urry C.M., Wamsteker W., Peracaula-Bosch M., 1992, ApJ 393,113

Clavel J., et al., 1991, ApJ 366, 64

Courvoisier T.J.-L., Clavel J., 1991, A\&A 248, 389 
Courvoisier T.J.-L., Paltani S., 1992, "IUE-ULDA Access Guide No. 4", Wamsteker W. (ed.) ESA SP 1153, p. 66

De Jong T., Clegg P.E., Soifer B.T., et al., 1984, ApJL 278, L67

De Martino D., Wamsteker W., Mas-Hesse J.M., Bonnell J., Imhoff C., 1991, ESA IUE Newslett. 38, 10

Edelson R.A., Malkan M.A., 1986, ApJ 308, 59

Edelson R.A., Malkan M.A., 1987, ApJ 323, 516

Edelson R.A., Krolik J.H., 1988, ApJ 333, 646

Fälker J., Gordon F., Sandford M.C.W., 1989, in "Exploring the Universe with the IUE satellite", Kondo Y. (ed.) Astrophys. Space Sci. Library, p. 21

Fairall A.P., 1977, MNRAS 180, 391

Ferland G.J., Osterbrock D.E., 1986, ApJ 300, 658

Ferland G.J., Korista K.T., Peterson B.M., 1990, ApJ 363, L21

Ferland G.J., 1991, "HAZY, a brief introduction to CLOUDY 80.06", Ohio State University Astr. Dpt., Internal Report 91-01, August 4

Gaskell C.M., Peterson B.M., 1987, ApJ 65, 1

Glass I.S., 1986, MNRAS 219, 5P

Griersmith D., Visvanathan N., 1979, A\&A 79, 329

Horne K., Welsh W.F., Peterson B.M., 1991, ApJ. Lett. 367, L5

Imhoff C.L, Wasatonic R., 1986, NASA IUE Newslett. 29, 45

Johnson H.L., 1966, ARA\&A 4, 193

Kollatschny W., Fricke K.J., 1985, A\&A Lett. 146, L11

Koratkar A.P., Gaskell C.M., 1989, ApJ 345, 637

Koratkar A.P., Gaskell C.M., 1991, ApJSS 75, 719

Kwan J., Krolik J.H., 1981, ApJ 250, 478

Lub J., Pel J.W., 1977, A\&A 54, 137

Lub J., de Ruiter H.R., 1992, A\&A 256, 33

Martin P.G., Thompson I.B., Maza J., Angel J.R.P., 1983, ApJ 266,470

Morini M., Scarsi L., Molteni D., et al., 1986, ApJ 307, 486

O'Brien P.T., Zheng W., 1990, Astrophys. Space Sci. 171, 77
Pelat D., Alloin D., Fosbury R.A.E., 1981, MNRAS 195, 787

Pérez E., Robinson A., De la Fuente L., 1992, MNRAS 255, 502

Pérez M., Loomis C., Eaton N., Bradley R., 1991, IUE 3Agency Meeting, June, p. VI-1

Pérez M., 1991, ESA IUE Newslett. 38, 27

Perola G.C., Piro L., Altamore A., Fiore F., 1986, ApJ 306, 508

Press W.H., Teukolsky S.A., 1988, Computers in Physics Nov./Dec., p. 77

Rieke G.H., Lebofsky M.J., 1985, ApJ 288, 618

Robinson A., Pérez E., 1990, MNRAS 244, 138

Rodríguez-Pascual P.M., 1989, Thesis, Universidad de Granada, Facultad de Ciencias

Seaton M.J., 1979, MNRAS 187, 73P

Shields J.C., Ferland G.J., Peterson B.M., 1995, ApJ 441, 507

Sonneborn G., Garhart M.P., 1987, ESA IUE Newslett. 28, 55

Véron-Cetty M.P., Woltjer L., Roy A.L., 1991, A\&A 246, L73

Vio R., Horne K., Wamsteker W., 1994, PASP 106, 1091

Wamsteker W., Alloin D., Pelat D., Gilmozzi R., 1985, ApJ 295, L33

Wamsteker W., Colina L., 1986, ApJ 311, 617

Wamsteker W., Rodríguez-Pascual P.M., Wills B.J., et al., 1990, ApJ 354, 446

Walter R., Orr A., Courvoisier T.J.-L., Fink H.H., Makino F., Wamsteker W., 1995, A\&A 438, 120

Ward M.J., Wilson A.S., 1978, ApJ 223, 788

West R.M., Danks A.C., Alcaino G., 1978, A\&A 62, L13

Whittle M., 1985, MNRAS 213, 1

Wills B.J., Netzer H., Wills D., 1985, ApJ 288, 94

Zdziarski A.A., Ghisellini G., George I.M., Svensson R., Fabian A.C., Done C., 1990, ApJ 363, L1

Zheng W., O'Brien P.T., 1990, ApJ 356, 463

Zheng W., Veilleux S., Grandi S.A., 1991, ApJ 381, 418 